\title{
Color-kinematics duality and double-copy construction for amplitudes from higher-dimension operators
}

\author{
Johannes Broedel ${ }^{a}$ and Lance J. Dixon ${ }^{b}$ \\ ${ }^{a}$ Stanford Institute for Theoretical Physics and Department of Physics, Stanford University, \\ Stanford, CA 94305, U.S.A. \\ ${ }^{b}$ SLAC National Accelerator Laboratory, Stanford University, \\ Stanford, CA 94309, U.S.A. \\ E-mail: jbroedel@ethz.ch, lance@slac.stanford.edu
}

ABSTRACT: We investigate color-kinematics duality for gauge-theory amplitudes produced by the pure nonabelian Yang-Mills action deformed by higher-dimension operators. For the operator denoted by $F^{3}$, the product of three field strengths, the existence of colorkinematic dual representations follows from string-theory monodromy relations. We provide explicit dual representations, and show how the double-copy construction of gravity amplitudes based on them is consistent with the Kawai-Lewellen-Tye relations. It leads to the amplitudes produced by Einstein gravity coupled to a dilaton field $\phi$, and deformed by operators of the form $\phi R^{2}$ and $R^{3}$. For operators with higher dimensions than $F^{3}$, such as $F^{4}$-type operators appearing at the next order in the low-energy expansion of bosonic and superstring theory, the situation is more complex. The color structure of some of the $F^{4}$ operators is incompatible with a simple color-kinematics duality based on structure constants $f^{a b c}$, but even the color-compatible $F^{4}$ operators do not admit the duality. In contrast, the next term in the $\alpha^{\prime}$ expansion of the superstring effective action - a particular linear combination of $D^{2} F^{4}$ and $F^{5}$-type operators - does admit the duality, at least for amplitudes with up to six external gluons.

KEYWORDS: Gauge-gravity correspondence, Scattering Amplitudes

ARXIV EPRINT: 1208.0876 


\section{Contents}

1 Introduction 1

2 Background $\quad 5$

2.1 Color-kinematics duality 5

2.2 Kawai-Lewellen-Tye relations 8

2.3 Double-copy construction of gravity amplitudes 9

$\begin{array}{llr}3 & F^{3} \text { modification of gauge theory } & 9\end{array}$

3.1 Known $F^{3}$ amplitudes 11

$\begin{array}{lll}3.2 & \text { CSW rules for } F^{3} \text { amplitudes } & 12\end{array}$

$\begin{array}{lll}3.3 & \text { Color-kinematics duality for } F^{3} \text { amplitudes } & 15\end{array}$

$\begin{array}{lll}3.4 & \text { Recycling of numerators } & 15\end{array}$

$\begin{array}{ll}\text { 3.5 } & \text { Monodromy relations and } F^{3} \text { amplitudes } \\ \end{array}$

4 Squaring to gravity $\quad 19$

$\begin{array}{ll}\text { 4.1 Set of amplitudes } & 19\end{array}$

$\begin{array}{lll}4.2 & \text { Consistency checks } & 20\end{array}$

4.3 KLT relations and effective actions for the bosonic string 22

$\begin{array}{llr}5 & \text { Beyond } F^{3} & 27\end{array}$

6 Conclusions and outlook 33

\section{Introduction}

In the 1980s, Kawai, Lewellen and Tye (KLT) [1] found a relation between tree-level open and closed string tree-level amplitudes, which implies - after taking the field-theory limit - that the color-ordered tree-level scattering amplitudes for gluons in Yang-Mills (YM) theories contain all the information necessary to construct the tree-level graviton scattering amplitudes in suitable gravity theories. More recently, Bern, Carrasco and Johansson (BCJ) [2] discovered that it is possible to directly construct gravity amplitudes from gaugetheory amplitudes, if the latter can be organized so that their numerator factors respect a certain color-kinematics duality. This method for obtaining gravity amplitudes is known as the double-copy construction. Color-kinematics duality is associated with linear BCJ relations between color-ordered subamplitudes. The BCJ amplitude relations have been proven using string-theory monodromy relations [3-5] and directly in field theory [6] using the BCFW recursion relations $[7,8]$. Given a color-kinematic dual representation for gauge theory, the double-copy construction has been proven to hold for all tree-level gravity amplitudes by Bern, Dennen, Huang, and Kiermaier [9]. Furthermore, in contrast to the 
field-theory limit of the KLT relations, the double-copy construction has been conjectured to hold for integrands of loop-level amplitudes as well. This conjecture has been verified, at different levels, in numerous examples [2, 10-16].

Color-kinematic dual representations exist for a variety of gauge-theory amplitudes. They have been shown to exist explicitly for tree-level amplitudes with up to eight external legs [2]. Expressions are also available for several loop-level integrands in maximally supersymmetric Yang-Mills (sYM) theory [2, 10, 11, 16], supporting the conjecture that all amplitudes of the theory can be cast into this form.

Gauge theories with fewer than the maximal number of supersymmetries are also expected to have dual representations, at both tree and loop level. At tree level, this assertion can often be demonstrated using the fact that the tree amplitudes coincide with the maximally supersymmetric case. At loop level, little has been shown explicitly to date (see, however, ref. [2] for a two-loop QCD example), primarily because the loop integrands for amplitudes in gauge theories with fewer supersymmetries are considerably more complicated than in the maximally supersymmetric case.

All of these discussions of color-kinematics duality are for amplitudes whose color decomposition can be expressed entirely in terms of the three-index antisymmetric Lie algebra structure constants $f^{a b c}$ for some nonabelian gauge group. Recently it has been pointed out that the notion of color-kinematics duality can be generalized to three-algebras, with a four-index antisymmetric structure constant $f^{a b c d}$ appearing in the trilinear product of generators, $\left[T^{a}, T^{b}, T^{c}\right]=f_{d}^{a b c} T^{d}[17]$. This algebra appears in the Bagger-LambertGustavsson theory [18-20], whose amplitudes exhibit this new color-kinematics duality. Ref. [17] also provides evidence for an associated double-copy construction of the amplitudes of maximal three-dimensional supergravity.

In four dimensions, gauge theories are renormalizable. However, they live in a larger class of nonrenormalizable theories, corresponding to the addition to the Lagrangian of a large variety of higher-dimension, gauge-invariant local operators built out of the gauge fields (and matter fields, if present). Some of these operators appear in the low-energy effective action of either bosonic or supersymmetric open string theory. In order to explore just how general the phenomenon of color-kinematics duality is, it is quite natural to ask whether the scattering amplitudes in these modified theories also have color-kinematic dual representations.

For pure gauge theory, the simplest candidate operator for modifying the YM action is the well-known operator $F^{3} \equiv \operatorname{Tr}\left(F_{\mu}{ }^{\nu} F_{\nu}{ }^{\rho} F_{\rho}{ }^{\mu}\right)$, where $F_{\mu \nu} \equiv F_{\mu \nu}^{a} T^{a}$ is the gluon field strength. This operator will be our prime example of a higher-dimension operator, but it is also special in several ways. It has dimension six, the lowest possible dimension for a modification of pure gauge theory. In the low-energy limit, or $\alpha^{\prime}$ expansion, of the effective action for the open bosonic string, the operator $F^{3}$ arises as the first correction to the usual YM Lagrangian $\operatorname{Tr}\left(F_{\mu \nu} F^{\mu \nu}\right)$. (See, for example, section 6.5 in ref. [21].)

The group theory structure implied by the $F^{3}$ modification mimics that of the YM action, in the sense that the three-gluon amplitudes in both cases are proportional to the structure constant $f^{a b c}$. In addition, the diagrams contributing to a particular amplitude are - up to changing the type of one vertex - topologically identical to the cubic graphs 
from pure YM-theory. Those two features suggest that color-kinematics duality might extend easily from amplitudes in YM theory to amplitudes produced by $F^{3}$. We use the string-theory monodromy relations $[3-5]$ to derive the BCJ relations between colorordered subamplitudes for $F^{3}$ amplitudes. We also compute a variety of $F^{3}$ amplitudes with different helicity structures and multiplicities, and verify the existence of color-kinematic dual representations.

In $\mathrm{QCD}$, the operator $F^{3}$ represents the only gauge-invariant modification of the three-gluon coupling which survives for on-shell three-point kinematics (with complex momenta) [22]. New physics, such as gluon compositeness or heavy colored states, could in principle produce this operator. There have been several phenomenological studies of its effects on high-energy collisions [23-28]. In pure-jet events at hadron colliders, the operator is difficult to observe, because at tree level it produces helicity amplitudes that are orthogonal to those of $\mathrm{QCD}$, and so the interference correction vanishes, until one reaches amplitudes with five external partons, corresponding to three-jet events [28]. At the threepoint level, QCD produces the (all-outgoing) helicity configurations $(--+)$ and $(++-)$, while the $F^{3}$ operator produces only $(---)$ and $(+++)$. At the four-point level, tree-level QCD produces only $(--++)$ (plus permutations), while $F^{3}$ produces $(----),(---+)$, $(+++-)$ and $(++++)$. In contrast, the four-point amplitudes involving a massive quark, such as $g g \rightarrow t \bar{t}$, do allow for a leading-order interference between the QCD amplitudes and those produced by the $F^{3}$ modification [23-25].

Several amplitudes for the scattering of massless partons using an $F^{3}$-modified action have been computed previously [28-30]. (Related amplitudes for a Higgs boson $H$ coupled to gluons via a heavy top-quark loop and the operator $H F^{3}$ have also been constructed recently [31].) In ref. [29] it was proposed to use a modification of the rules for pure gauge theory due to Cachazo, Svrček and Witten (CSW) [32], which are called CSW rules and employ maximally-helicity-violating (MHV) vertices. In ref. [33] these rules were proven, using canonical transformations, and even allowing for additional coupling of $F^{3}$ to a scalar (Higgs) field. Here we compute explicitly higher-multiplicity $F^{3}$ amplitudes requiring a number of MHV vertices, so that we can check their compatibility with colorkinematics duality.

Another purpose of this article is to investigate whether the double-copy construction for gravity can be extended to the case of gravity perturbed by the operators up to $R^{3}$ (a suitable contraction of three Riemann tensors), utilizing the amplitudes produced by $F^{3}$. We find evidence that it can, provided that intermediate exchanges of the dilaton/axion field of string theory are properly taken into account. The connection between gravity amplitudes from $R^{3}$ and gauge-theory amplitudes from $F^{3}$ via the KLT relations at the three- and four-point level was studied previously [34, 35]. It is simple to see that the three-point amplitudes are related by 'squaring'. Beyond the three-point level, however, one cannot avoid the introduction of the dilaton on the closed-string side in order to reproduce the corrections to the string amplitude. The $F^{3}$-modified amplitudes are order$\alpha^{\prime}$ corrections to gluon scattering in open bosonic string theory. When one applies a double-copy construction based on a pair of $F^{3}$-modified amplitudes, that corresponds to an order- $\alpha^{\prime 2}$ correction to graviton amplitudes in closed string theory. At order $\alpha^{\prime 2}$, the 
closed string effective action contains an operator of the form $R^{3}$. However, at order $\alpha^{\prime}$ it also contains an operator of the form $e^{-2 \phi} R^{2}$, which can emit a dilaton $\phi$. Two insertions of this operator will contribute to amplitudes where the external states are all gravitons - an intermediate dilaton is created and destroyed by the two operator insertions. For low-multiplicity amplitudes, we will demonstrate explicitly how the various contributions combine in the double-copy construction.

At higher multiplicity, the general arguments for the double-copy construction in Einstein gravity [9] rely on a color-kinematic dual representation of the gauge-theory amplitude. This representation is satisfied by all the $F^{3}$ amplitudes we have constructed. However, the double-copy arguments [9] also rely on deformations of both gravity and gauge-theory amplitudes by complex-momentum shifts $[7,8]$. Generic amplitudes perturbed by higher-dimension operators typically do not converge at large values of the shift. Therefore we do not have a general argument for a double-copy construction of the amplitudes produced by $R^{3}$ (plus $e^{-2 \phi} R^{2}$ ). On the other hand, we will provide evidence, for all helicities up to six external legs, in favor of the $R^{3}$ double-copy construction. For the case of identical-graviton helicities, we can use an all-line complex-momentum shift [30] to determine the $R^{3}$ amplitudes. We will also perform indirect tests of the validity of the double-copy construction, or alternatively a KLT construction of $R^{3}$ amplitudes, by testing their Bose symmetry under the exchange of identical-helicity gravitons, and their universal behavior in collinear and soft limits.

Returning to the gauge-theory Lagrangian, we consider the addition of yet higherdimension pure Yang-Mills operators (dimension eight or more). At dimension eight, there are four independent operators involving four gluon field strengths ( $F^{4}$-type operators). Two linear combinations of these four operators appear in the low-energy expansion of the open bosonic string and open superstring, respectively. The amplitudes generated by these two linear combinations of operators are readily available from string theory. They allow for the testing of possible color-kinematic dual representations. We find that the bosonic and superstring amplitudes individually correspond to operators whose color structure is incompatible with the usual color-kinematics duality, simply because they cannot be expressed in terms of structure constants $f^{a b c}$ alone. (In this paper we do not consider extensions of color-kinematics duality to four-index antisymmetric structure constants [17].) The difference between the bosonic string and superstring amplitudes actually has a colorcompatible representation, and even obeys a BCJ-like monodromy relation. However, we find that the corresponding operator does not admit a color-kinematic dual representation, for reasons we shall explain.

On the other hand, at the next order in $\alpha^{\prime}$, we find that the operator appearing at $\mathcal{O}\left(\alpha^{\prime 3}\right)$ in the superstring effective action is color-compatible, and its amplitudes do satisfy color-kinematics duality at the level of four, five and six external gluons. Beyond this order in $\alpha^{\prime}$, we tested the superstring four-point amplitudes and did not find any dual representations. This property can be explained simply by the fact that the color structures of the amplitudes at orders higher than $\mathcal{O}\left(\alpha^{\prime 3}\right)$ cannot be expressed in terms of structure constants $f^{a b c}$ alone.

The article is organized as follows. In section 2, we review color-kinematics duality and 
BCJ relations, as well as the KLT relations and the double-copy construction. Section 3 is devoted to the $F^{3}$-modified theory. It explains how the amplitudes in this theory can be computed using CSW or MHV rules, and provides evidence that they obey the duality. In section 4 , we discuss the double-copy construction for amplitudes produced by operators up to $R^{3}$ in gravity. In section 5 we discuss the insertion of operators in gauge theory with dimension greater than six, paying particular attention to their color structure. At dimension eight, we find no operators that are consistent with color-kinematics duality; but at dimension ten, the $\mathcal{O}\left(\alpha^{\prime 3}\right)$ term in the superstring effective action appears to be consistent. Finally, in section 6 we summarize our findings and point out avenues for future research.

\section{Background}

\subsection{Color-kinematics duality}

A fully color-dressed $m$-gluon tree amplitude in YM theory can be decomposed as

$$
\mathcal{A}_{m} \equiv \mathcal{A}_{m}\left(a_{1}, \ldots, a_{m}\right)=\sum_{\sigma \in S_{m} / Z_{m}} \operatorname{Tr}\left(T^{a_{\sigma(1)}} \ldots T^{a_{\sigma(m)}}\right) A_{m}(\sigma(1), \ldots, \sigma(m))
$$

where the $a_{i}$ are adjoint indices, the $A_{m}$ are color-ordered subamplitudes, and the sum is over non-cyclic permutations $S_{m} / Z_{m}=S_{m-1}$. We have set the gauge coupling $g$ to 1 for convenience. The trace runs over the color generators $T^{a}$, which are assumed to be in the fundamental representation of the gauge group. The generators are normalized to $\operatorname{Tr}\left(T^{a} T^{b}\right)=\delta^{a b}$, and the structure constants are defined by

$$
\tilde{f}^{a b c} \equiv i \sqrt{2} f^{a b c}=\operatorname{Tr}\left(\left[T^{a}, T^{b}\right] T^{c}\right) .
$$

Starting from the decomposition in eq. (2.1), it takes only a couple of steps to demonstrate the idea behind color-kinematic dual representations: the color and the kinematic part of the amplitude are brought into a form in which they behave identically under certain symmetry operations. As an example, we will perform the necessary steps on the four-point tree amplitude, which has the color decomposition,

$$
\begin{aligned}
\mathcal{A}_{4}\left(a_{1}, a_{2}, a_{3}, a_{4}\right)= & \sum_{\sigma \in S_{3}} \operatorname{Tr}(\sigma(1), \ldots, \sigma(4)) A_{4}(\sigma(1), \ldots, \sigma(4)) \\
= & \overleftrightarrow{\operatorname{Tr}}(1,2,3,4) A_{4}(1,2,3,4)+\overleftrightarrow{\operatorname{Tr}}(1,3,2,4) A_{4}(1,3,2,4) \\
& +\overleftrightarrow{\operatorname{Tr}}(1,2,4,3) A_{4}(1,2,4,3) .
\end{aligned}
$$

Here we have shortened the expression by using the reflection symmetry of the subamplitudes, and by writing $\operatorname{Tr}\left(T^{a_{i_{1}}} T^{a_{i_{2}}} T^{a_{i_{3}}} T^{a_{i_{4}}}\right)=\operatorname{Tr}\left(i_{1}, i_{2}, i_{3}, i_{4}\right)$ and $\operatorname{Tr}\left(i_{1}, i_{2}, i_{3}, i_{4}\right)+$ $\operatorname{Tr}\left(i_{4}, i_{3}, i_{2}, i_{1}\right)=\overleftrightarrow{\operatorname{Tr}}\left(i_{1}, i_{2}, i_{3}, i_{4}\right)$

Color-kinematic dual representations rely on the organization of amplitudes in terms of cubic graphs. For four external gluons, the three distinct cubic graphs shown in figure 1 contribute to the scattering amplitude. Each graph can be written as its color factor (here a product of two structure constants) multiplied by a kinematic factor of the form $\frac{\text { numerator }}{\text { propagator }}$, 


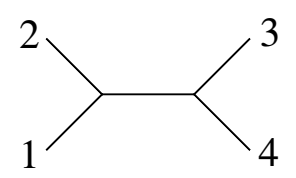

(s)

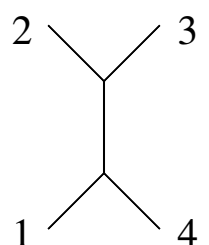

(t)

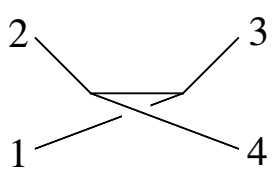

(u)

Figure 1. The three cubic graphs at the four-point level.

which here takes the form, $\frac{n_{s}}{s}, \frac{n_{t}}{t}$ and $\frac{n_{u}}{u}$. The Mandelstam variables appropriate for the four-point amplitude with external momenta $k_{1}, k_{2}, k_{3}, k_{4}$ are $s=\left(k_{1}+k_{2}\right)^{2}, t=\left(k_{2}+k_{3}\right)^{2}$ and $u=\left(k_{1}+k_{3}\right)^{2}$. The numerator factors $n_{i}$ are labeled by the channel, $s, t$ or $u$.

In a cubic-graph representation, the diagram produced by the four-gluon contact interaction, which is proportional to the product of two structure constants, has already been absorbed by expressing its kinematic part using inverse propagators in the numerator, to compensate for the propagator denominators. (A similar construction is possible for other four-point contact interactions, provided that they have the correct type of color structure.)

Each color-ordered amplitude can now be expressed in terms of two cubic graphs. For example, the first two graphs in figure 1 contribute to $A_{4}(1,2,3,4)$,

$$
A_{4}(1,2,3,4)=\frac{n_{s}}{s}+\frac{n_{t}}{t} .
$$

Employing in addition the antisymmetry of the cubic vertices, which implies, for instance,

$$
n_{s}(2,1,3,4)=-n_{s}(1,2,3,4),
$$

we can rewrite eq. (2.3) as

$$
\begin{aligned}
\mathcal{A}_{4}\left(a_{1}, a_{2}, a_{3}, a_{4}\right)= & {[\overleftrightarrow{\operatorname{Tr}}(1,2,3,4)-\overleftrightarrow{\operatorname{Tr}}(1,2,4,3)] \frac{n_{s}}{s}+[\overleftrightarrow{\operatorname{Tr}}(1,2,3,4)-\overleftrightarrow{\operatorname{Tr}}(1,3,2,4)] \frac{n_{t}}{t} } \\
& +[\overleftrightarrow{\operatorname{Tr}}(1,3,2,4)-\overleftrightarrow{\operatorname{Tr}}(1,2,4,3)] \frac{n_{u}}{u} .
\end{aligned}
$$

Finally, one can convert the combination of traces into structure constants using: ${ }^{1}$

$$
\overleftrightarrow{\operatorname{Tr}}(1,2,3,4)-\overleftrightarrow{\operatorname{Tr}}(1,2,4,3)=\tilde{f}^{a_{1} a_{2} e} \tilde{f}^{e a_{3} a_{4}}
$$

Thus, the full color-dressed four-point tree amplitude can be represented as

$$
\mathcal{A}_{4}\left(a_{1}, a_{2}, a_{3}, a_{4}\right)=\frac{\tilde{f}^{a_{1} a_{2} e} \tilde{f}^{e a_{3} a_{4}} n_{s}}{s}+\frac{\tilde{f}^{a_{2} a_{3} e} \tilde{f}^{e a_{4} a_{1}} n_{t}}{t}+\frac{\tilde{f}^{a_{1} a_{3} e} \tilde{f}^{e a_{2} a_{4}} n_{u}}{u}=\sum_{g \in \Gamma_{4}} \frac{c_{g} n_{g}}{p_{g}^{2}}
$$

where $\Gamma_{4}$ is the set of cubic tree graphs in figure $1 ; c_{g}$ and $n_{g}$ are mappings providing, respectively, the color and numerator factors of the graph $g$; and $1 / p_{g}^{2}$ denotes the scalar propagator of the graph. Color-kinematics duality for four-point amplitudes was first identified in refs. [37, 38].

\footnotetext{
${ }^{1}$ This method is the guiding idea behind establishing a dual representation of amplitudes in ref. [36].
} 
For a general $m$-point scattering amplitude at tree level, the correspondence between color and kinematics [2] begins with the cubic-graph representation,

$$
\mathcal{A}_{m}=\sum_{g \in \Gamma_{m}} \frac{c_{g} n_{g}}{\prod_{l \in P_{g}} p_{l}^{2}},
$$

where the sum runs over all $m$-point cubic tree graphs $\Gamma_{m}$. The product in the denominator runs over the set $P_{g}$ of all scalar propagators $l$, with momenta $p_{l}$, that appear in the graph $g$. In addition, we have omitted the Yang-Mills coupling constant for convenience.

Color-kinematics duality holds if the kinematic factors obey two criteria:

1. For a pair of graphs $g$ and $\hat{g}$ that are related by an exchange of two external legs connected by a three-vertex, the color factors are related by the antisymmetry of the structure constant, $c_{g}=-c_{\hat{g}}$. The numerator factors must also be antisymmetric,

$$
n_{g}=-n_{\hat{g}}
$$

2. For a triplet of graphs $i, j, k$ that differ only by the routing of lines in a four-point subgraph, as in figure 1 , the color Jacobi relations imply that $c_{i}+c_{j}+c_{k}=0$. Then the numerators must obey

$$
n_{i}+n_{j}+n_{k}=0 .
$$

There are (coupled) sign ambiguities in defining the $c_{g}$ and $n_{g}$; hence other presentations are possible, such as $c_{i}=c_{j}+c_{k}$ accompanied by $n_{i}=n_{j}+n_{k}$.

A set of numerators $n_{g}$ satisfying eqs. (2.10) and (2.11) is called a color-kinematic dual representation.

In the four-point example (2.8), the one Jacobi constraint on the numerators is

$$
n_{s}=n_{t}+n_{u},
$$

corresponding to the Jacobi relation

$$
\tilde{f}^{a_{1} a_{2} e} \tilde{f}^{e a_{3} a_{4}}=\tilde{f}^{a_{2} a_{3} e} \tilde{f}^{e a_{4} a_{1}}+\tilde{f}^{a_{1} a_{3} e} \tilde{f}^{e a_{2} a_{4}} .
$$

In the general case, the constraints (2.11) are very powerful, considerably reducing the number of independent color-ordered subamplitudes. Naively, there are $(m-1)$ ! independent subamplitudes in eq. (2.1), corresponding to the number of cyclically inequivalent traces. This number is reduced to $(m-2)$ ! by the group-theoretic Kleiss-Kuijf relations [39, 40]. The linear relations that follow from consistency with eqs. (2.11) are known as the BCJ amplitude relations [2-5]. They further reduce the number of independent subamplitudes to $(m-3)$ !.

At the four-point level, there is only one BCJ relation,

$$
0=s A_{4}(2,1,3,4)-t A_{4}(2,3,1,4) .
$$

It reduces the two Kleiss-Kuijf independent subamplitudes down to a single independent subamplitude. 
Although the existence of BCJ relations is intimately tied to the existence of colorkinematic dual representations, there is no proof of equivalence for generic theories including nonrenormalizable operators. A color-kinematic dual representation does imply the existence of BCJ amplitude relations. Concerning the reverse direction, explicit (albeit non-local) representations of dual numerators $n_{g}$ in terms of color-ordered amplitudes have been obtained for any number of external legs in (renormalizable) gauge theory, based on string theory arguments [41-43]. For MHV amplitudes, a construction of dual numerators has been given based on the existence of a diffeomorphism Lie algebra for the self-dual theory $[44,45]$. Below, we will test for color-kinematic dual representations of $F^{3}$ amplitudes by explicitly constructing or calculating the numerators. At the same time we will also test the BCJ amplitude relations explicitly, and provide a general argument for their validity. These results further support the equivalence of BCJ relations and color-kinematic dual representations, at least in the $F^{3}$ case.

\subsection{Kawai-Lewellen-Tye relations}

Tree-level amplitudes in closed and open string theories are linked by the KLT relations [1], which arise from the fact that any closed-string vertex operator can be represented as a product of two open-string vertex operators. Closed-string scattering amplitudes are sums of products of left-moving and right-moving open-string scattering amplitudes, multiplied by various sine functions of the kinematics arising from contour deformations. When one takes the low-energy, or infinite string tension $\left(\alpha^{\prime} \rightarrow 0\right)$, limit of the KLT relations, the sine functions collapse to Mandelstam variables, and the relations express gravity amplitudes $M_{m}$ in terms of color-ordered amplitudes $A_{m}$ in gauge theory. For three to six external legs, the field-theory KLT relations are given by,

$$
\begin{aligned}
M_{3}(1,2,3)= & i A_{3}(1,2,3) \tilde{A}_{3}(1,2,3), \\
M_{4}(1,2,3,4)= & -i s_{12} A_{4}(1,2,3,4) \tilde{A}_{4}(1,2,4,3), \\
M_{5}(1,2,3,4,5)= & i s_{12} s_{34} A_{5}(1,2,3,4,5) \tilde{A}_{5}(2,1,4,3,5)+\mathcal{P}(2,3), \\
M_{6}(1,2,3,4,5,6)= & -i s_{12} s_{45} A_{6}(1,2,3,4,5,6) \\
& \times\left[s_{35} \tilde{A}_{6}(2,1,5,3,4,6)+\left(s_{34}+s_{35}\right) \tilde{A}_{6}(2,1,5,4,3,6)\right] \\
& +\mathcal{P}(2,3,4),
\end{aligned}
$$

where $s_{i j}=\left(k_{i}+k_{j}\right)^{2}$. Formulae for higher-point amplitudes can be derived straightforwardly [1]. In the above equation, ' $+\mathcal{P}$ ' indicates a sum over all $p$ ! nontrivial permutations of the $p$ indicated indices, acting on the term shown. We refer to the amplitudes $A_{m}\left(\tilde{A}_{m}\right)$ as coming from a 'left-moving' ('right-moving') gauge theory. In general, we may write the all-multiplicity KLT relations in the field theory limit as a bilinear functional of the gauge-theory amplitudes,

$$
M_{m}=\operatorname{KLT}_{\mathrm{FT}, m}\left[A_{m}, \tilde{A}_{m}\right] .
$$

The definitions of $\mathrm{KLT}_{\mathrm{FT}, m}$ for $m=3,4,5,6$ follow from eqs. (2.15). For general $m$, the definitions follow from the explicit formulae available in ref. [46]. Below, we will use the notation in eq. (2.16) in order to describe extensions of the field-theory KLT relations to cover amplitudes generated by higher-dimension operators. 


\subsection{Double-copy construction of gravity amplitudes}

The field-theory limit of the KLT relations, combined with the unitarity method [47, 48], has provided, until recently, the most important technical tool for perturbative calculations in maximal $\mathcal{N}=8$ supergravity. Multi-loop $\mathcal{N}=8$ supergravity integrands can be constructed by matching them against generalized unitarity cuts, which are computed using products of $\mathcal{N}=8$ supergravity tree amplitudes. The tree-amplitude products can be evaluated efficiently using the KLT relations, together with the generalized cuts of corresponding multi-loop amplitudes in $\mathcal{N}=4$ super-Yang-Mills theory [49-51]. However, this method still requires some effort, namely to process the generalized cut information into a local gravity integrand.

In contrast, once an explicit color-kinematic dual representation is found for a certain scattering amplitude in gauge theory, the double-copy construction $[2,9,10]$ dramatically simplifies the construction of the corresponding amplitude in the gravity theory. According to this construction, one simply replaces the color factors $c_{g}$ in eq. (2.9) with a second set of gauge-theory kinematical numerators $\tilde{n}_{g}$, while keeping the remainder of the expression untouched. (The $\tilde{n}_{g}$ numerators come from the same right-moving gauge theory discussed earlier in the context of the KLT relations.) This method works at both the tree and loop level $[2,9]$. The tree-level version, starting from eq. (2.9), is

$$
M_{m}=i \sum_{g \in \Gamma_{m}} \frac{n_{g} \tilde{n}_{g}}{\prod_{l \in P_{g}} p_{l}^{2}} .
$$

For convenience, we have omitted overall factors of $(\kappa / 2)$, where $\kappa$ is the gravitational coupling.

While the KLT relations pertain exclusively to tree amplitudes, the BCJ-inspired double-copy construction has been conjectured to hold to all loop orders. It has been demonstrated to work in many examples $[11,13]$. Indeed, it has been used to obtain threeand four-loop integrands in $\mathcal{N}=8$ supergravity that make manifest, term by term, the correct ultraviolet behavior of the full amplitude [10, 16].

In the next section, we will provide strong evidence that the gauge-theory amplitudes produced by adding the operator $F^{3}$ to the action also have a color-kinematic dual representation. In the section after that, we will use this property to generate a double-copy construction of the gravitational amplitudes produced by the operators $e^{-2 \phi} R^{2}$ and $R^{3}$ in the effective action of the closed bosonic string.

\section{$3 \quad F^{3}$ modification of gauge theory}

In this section we consider the following addition to the YM action,

$$
O_{\Lambda}=\frac{1}{\Lambda^{2}} \operatorname{Tr}\left(F_{\mu}{ }^{\nu} F_{\nu}{ }^{\rho} F_{\rho}{ }^{\mu}\right) \text {. }
$$

This operator, referred to as $F^{3}$, is the unique, gauge-invariant, CP-even dimension-six operator built exclusively from gluon fields. The prefactor $1 / \Lambda^{2}$, where $\Lambda$ has mass-dimension one, renders the operator four-dimensional. 
The antisymmetry of the gauge field strength, $F_{\rho \mu}=-F_{\mu \rho}$, enables us to immediately rewrite the color trace as

$$
\operatorname{Tr}\left(F_{\mu}{ }^{\nu} F_{\nu}{ }^{\rho} F_{\rho}{ }^{\mu}\right)=\frac{1}{2} \operatorname{Tr}\left(\left[T^{a}, T^{b}\right] T^{c}\right) F_{\mu}^{a \nu} F_{\nu}^{b \rho} F_{\rho}^{c \mu}=\frac{1}{2} \tilde{f}^{a b c} F_{\mu}^{a \nu} F_{\nu}^{b \rho} F_{\rho}^{c \mu} .
$$

Because the symmetries of the kinematic part of this operator project onto the totally antisymmetric part of the trace, the resulting $\tilde{f}^{a b c}$ color structure for the three-vertex generated by $F^{3}$ is the same as in usual YM theory.

In this article we will mostly consider amplitudes whose deviation from the pure YM action is linear in $1 / \Lambda^{2}$. These amplitudes arise from one insertion of the $F^{3}$ vertex, combined with any number of tree-level YM interactions. The only exception occurs in section 5 , where we need to consider a diagram with two insertions of $F^{3}$ vertices, in order to explain the difference between bosonic string theory and superstring theory at second order in the inverse string tension.

One can decompose the $F^{3}$ insertion into a holomorphic (self-dual) and an antiholomorphic (anti-self-dual) part,

$$
\begin{aligned}
O_{\Lambda} & =O_{\Lambda_{+}}+O_{\Lambda_{-}} \\
O_{\Lambda_{+}} & =\frac{1}{\Lambda^{2}} \operatorname{Tr}\left(F_{\mathrm{SD} \mu}^{\nu} F_{\mathrm{SD} \nu}^{\rho} F_{\mathrm{SD} \rho}^{\mu}\right) \equiv F_{\mathrm{SD}}^{3}, \\
O_{\Lambda_{-}} & =\frac{1}{\Lambda^{2}} \operatorname{Tr}\left(F_{\mathrm{ASD} \mu}^{\nu} F_{\mathrm{ASD} \nu}^{\rho} F_{\mathrm{ASD} \rho}^{\mu}\right) \equiv F_{\mathrm{ASD}}^{3}
\end{aligned}
$$

where

$$
F_{\mathrm{SD}}^{\mu \nu}=\frac{1}{2}\left(F^{\mu \nu}+\tilde{F}^{\mu \nu}\right), \quad F_{\mathrm{ASD}}^{\mu \nu}=\frac{1}{2}\left(F^{\mu \nu}-\tilde{F}^{\mu \nu}\right), \quad \tilde{F}^{\mu \nu} \equiv \frac{i}{2} \epsilon^{\mu \nu \rho \sigma} F_{\rho \sigma} .
$$

This decomposition exposes the MHV structure of the amplitudes produced by $F^{3}$ [29]. Parity exchanges the two operators, $O_{\Lambda_{+}} \leftrightarrow O_{\Lambda_{-}}$.

Combining one vertex from $F_{\mathrm{SD}}^{3}$ with an arbitrary number of pure YM vertices leads to all the amplitudes in the left tower in figure 2 (red dots). All amplitudes from the right tower (green dots), corresponding to amplitudes originating from the anti-self-dual operator $F_{\mathrm{ASD}}^{3}$, can be obtained immediately from the left tower using parity. Parity exchanges positive- and negative-helicity gluons, reflecting across the vertical axis in the figure, and also complex conjugates all spinors. The amplitudes produced by $F^{3}$ in the overlapping region can be obtained by adding the self-dual and anti-self-dual contributions. We denote the amplitudes produced by $F^{3}, F_{\mathrm{SD}}^{3}$ and $F_{\mathrm{ASD}}^{3}$, respectively, by $A_{m}^{F}, A_{m}^{F+}$ and $A_{m}^{F-}$, with $A_{m}^{F}=A_{m}^{F+}+A_{m}^{F-}$.

From now on, unless otherwise specified, we consider the left tower, namely the amplitudes $A_{m}^{F+}$ produced by $F_{\mathrm{SD}}^{3}$. Every nonvanishing amplitude of this type should have at least three negative-helicity gluons [29]. That is,

$$
A_{m}^{F+}\left(1^{+}, \ldots, m^{+}\right)=A_{m}^{F+}\left(1^{+}, \ldots, j^{-}, \ldots, m^{+}\right)=A_{m}^{F+}\left(1^{+}, \ldots, j^{-}, \ldots, k^{-}, \ldots, m^{+}\right)=0
$$

where $j$ and $k$ are the only negative-helicity gluons. Amplitudes with exactly three negativehelicity gluons and an arbitrary number of positive-helicity gluons are called MHV amplitudes. (In the right tower generated by $F_{\mathrm{ASD}}^{3}$, the amplitudes with exactly three positive- 


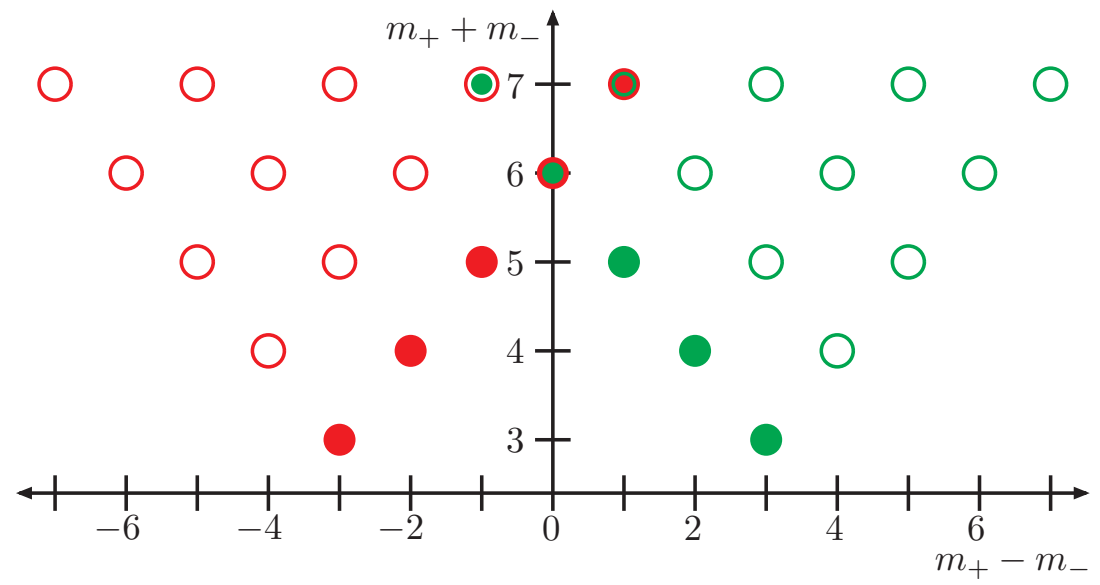

Figure 2. The $m$-gluon amplitudes produced by the operator $F^{3}$, where $m_{+}$and $m_{-}$are the numbers of positive- and negative-helicity gluons, respectively. The left tower (red circles) of amplitudes is the self-dual sector, produced by $F_{\mathrm{SD}}^{3}$. The right tower (green circles) is produced by the anti-self-dual operator $F_{\mathrm{ASD}}^{3}$. For MHV $(\overline{\mathrm{MHV}})$ amplitudes with exactly three negative (positive) helicities, the circles are filled. The figure is from ref. [29].

helicity gluons, and the rest negative, form the $\overline{\mathrm{MHV}}$ class.) The lowest interaction derivable from the self-dual part belongs to the MHV class and is a three-point vertex with three negative helicities,

$$
A_{3}^{F+}\left(1^{-}, 2^{-}, 3^{-}\right)=i\langle 12\rangle\langle 23\rangle\langle 31\rangle
$$

It is not an on-shell amplitude for real momenta, but it is perfectly well-defined and nonzero for complex momenta. In writing the amplitudes $A_{m}^{F}, A_{m}^{F+}$ and $A_{m}^{F-}$, we omit for convenience an overall factor of $-3 g^{m-2} / \Lambda^{2}$.

\subsection{Known $F^{3}$ amplitudes}

The Parke-Taylor formula [52] for MHV tree amplitudes in YM theory reads,

$$
A_{m}\left(1^{+}, \ldots, j^{-}, \ldots, k^{-}, \ldots, m^{+}\right)=i \frac{\langle j k\rangle^{4}}{\langle 12\rangle\langle 23\rangle \cdots\langle m 1\rangle}
$$

where $j$ and $k$ are the only negative-helicity gluons, and the usual spinor-helicity brackets $\langle j k\rangle$ have been used. Analogous expressions for the MHV amplitudes in the $F^{3}$ sector, $A_{m}^{F+}$, were first obtained for $m=4,5[28]$, and were later generalized to other values of $m$ [29]. They take the form,

$$
A_{m}^{F+}\left(1^{+}, \ldots, j^{-}, \ldots, k^{-}, \ldots, l^{-}, \ldots, m^{+}\right)=i \frac{\langle j k\rangle^{2}\langle k l\rangle^{2}\langle l j\rangle^{2}}{\langle 12\rangle\langle 23\rangle \cdots\langle m 1\rangle},
$$

where $j, k$ and $l$ are the only negative-helicity gluons.

Besides the MHV amplitudes in eq. (3.9), several further four-parton amplitudes have been computed $[28,29]$. At the four-point level (and three-point level) the $F^{3}$ amplitudes 


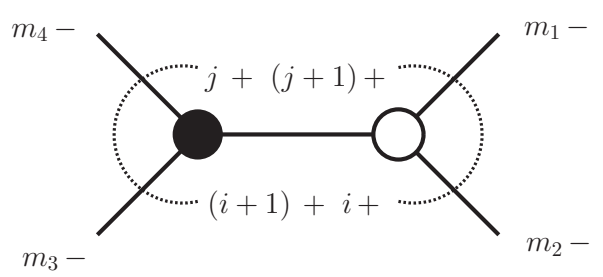

(a)

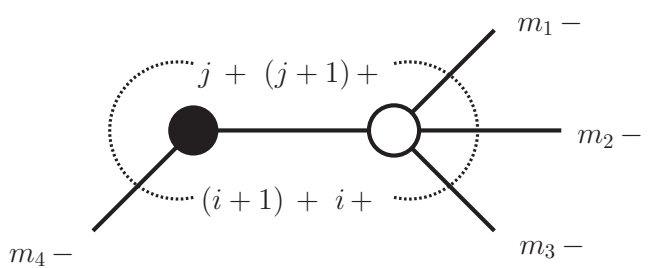

(b)

Figure 3. Next-to-MHV $F^{3}$ amplitudes are constructed by sewing together two MHV vertices, one for $F^{3}$ (white circle) and one for ordinary gauge theory (black dot). There are two distinct ways of distributing the four negative-helicity gluons, (a) and (b). The dotted lines indicate that an arbitrary number of positive-helicity gluons may be present.

are orthogonal to the pure YM amplitudes. The only non-vanishing, non-MHV four-point amplitude produced by $F_{\mathrm{SD}}^{3}$ is

$$
A_{4}^{F+}\left(1^{-}, 2^{-}, 3^{-}, 4^{-}\right)=\frac{-2 i s_{12} s_{23} s_{13}}{[12][23][34][41]}
$$

which has a very simple, cyclicly symmetric form.

\subsection{CSW rules for $F^{3}$ amplitudes}

In order to generate further $F^{3}$ amplitudes, beyond those given above, we adapt the CSW formalism [32] based on MHV vertices to the present context [29, 33]. An $\mathrm{N}^{k} \mathrm{MHV}$ amplitude $A_{m}^{F+}$ is generated by precisely one $F_{\mathrm{SD}}^{3}$ MHV vertex (the off-shell continuation of eq. (3.9)) and $k$ YM-theory MHV vertices (the off-shell continuation of the Parke-Taylor formula (3.8)). Because the $F_{\mathrm{SD}}^{3} \mathrm{MHV}$ vertex provides three negative helicities, not two, an $\mathrm{N}^{k} \mathrm{MHV}$ amplitude contains $k+3$ negative-helicity gluons. The prescription for continuing off shell a holomorphic (right-handed) spinor $\lambda_{a}$ that enters a CSW propagator is exactly as in YM theory. Namely, one introduces a reference spinor $\xi$ via the replacement

$$
\left(\lambda_{a}\right)_{\alpha} \rightarrow\left(p_{a}\right)_{\alpha \dot{\alpha}} \xi^{\dot{\alpha}}
$$

whenever leg $a$ is off shell.

At next-to-MHV (NMHV), the two distinct classes of MHV-vertex graphs are shown in figure 3. Following refs. [29, 53], they generate the amplitudes

$$
\begin{aligned}
& A_{m}^{F+}\left(m_{1}^{-}, m_{2}^{-}, m_{3}^{-}, m_{4}^{-}\right) \\
& \quad=i \frac{\sum_{C\left(m_{1}, m_{2}, m_{3}, m_{4}\right)}\left[A_{m}^{(a)}\left(m_{1}, m_{2}, m_{3}, m_{4}\right)+A_{m}^{(b)}\left(m_{1}, m_{2}, m_{3}, m_{4}\right)\right]}{\prod_{l=1}^{m}\langle l, l+1\rangle}
\end{aligned}
$$

where we have omitted the labels of the $(m-4)$ positive-helicity gluons. The sum over $C\left(m_{1}, m_{2}, m_{3}, m_{4}\right)$ is over the four cyclic permutations of the four negative-helicity labels. 
The two distinct classes of graphs evaluate to

$$
\begin{aligned}
& A_{m}^{(a)}\left(m_{1}, m_{2}, m_{3}, m_{4}\right)=\sum_{i=m_{2}} \sum_{j=m_{4}}^{m_{3}-1} \frac{\left\langle m_{1} m_{2}\right\rangle^{2}\left\langle m_{1}^{-}\left|\phi_{j+1, i}\right| \xi^{-}\right\rangle^{2}\left\langle m_{2}^{-}\left|\phi_{j+1, i}\right| \xi^{-}\right\rangle^{2}\left\langle m_{3} m_{4}\right\rangle^{4}}{D\left(i, j, q_{j+1, i}\right)} \\
& A_{m}^{(b)}\left(m_{1}, m_{2}, m_{3}, m_{4}\right)=\sum_{i=m_{3}}^{m_{4}-1} \sum_{j=m_{4}}^{m_{1}-1} \frac{\left\langle m_{1} m_{2}\right\rangle^{2}\left\langle m_{2} m_{3}\right\rangle^{2}\left\langle m_{3} m_{1}\right\rangle^{2}\left\langle m_{4}^{-}\left|\phi_{j+1, i}\right| \xi^{-}\right\rangle^{4}}{D\left(i, j, q_{j+1, i}\right)}
\end{aligned}
$$

where $q_{j+1, i}=k_{j+1}+k_{j+2}+\cdots+k_{i}$, and all external leg labels are defined modulo $m$. The 'dressed' propagator is,

$$
D(i, j, q)=\left\langle i^{-}|q| \xi^{-}\right\rangle\left\langle(i+1)^{-}|\not| \xi^{-}\right\rangle\left\langle j^{-}|\not| \xi^{-}\right\rangle\left\langle(j+1)^{-}|q| \xi^{-}\right\rangle \frac{q^{2}}{\langle i, i+1\rangle\langle j, j+1\rangle} .
$$

The sum over $i, j$ is over the ways of distributing the positive-helicity gluons. In figure $3(\mathrm{~b})$ there must be at least one positive-helicity gluon attached to the left vertex. If there is not, the factor $\left\langle m_{4}^{-}\left|\phi_{j+1, i}\right| \xi^{-}\right\rangle=-\left\langle m_{4}^{-}\left|k_{m_{4}}\right| \xi^{-}\right\rangle=0$ kills the corresponding term in any event. We have also implemented the general formula for $\mathrm{N}^{2} \mathrm{MHV}$ amplitudes, which involves 14 distinct classes of CSW diagrams. Finally, we worked out the one $\mathrm{N}^{3} \mathrm{MHV}$ amplitude at the six-point level, $A_{6}^{F+}$ with all negative helicities, in terms of 56 CSW diagrams.

Employing this modified CSW procedure, we have calculated the following amplitudes:

$$
\begin{aligned}
& A_{5}^{F+}\left(1^{-}, 2^{-}, 3^{-}, 4^{-}, 5^{+}\right), \\
& A_{5}^{F+}\left(1^{-}, 2^{-}, 3^{-}, 4^{-}, 5^{-}\right), \\
& A_{6}^{F+}\left(1^{-}, 2^{-}, 3^{-}, 4^{-}, 5^{+}, 6^{+}\right), \\
& A_{6}^{F+}\left(1^{-}, 2^{-}, 3^{-}, 4^{+}, 5^{-}, 6^{+}\right), \\
& A_{6}^{F+}\left(1^{-}, 2^{-}, 3^{+}, 4^{-}, 5^{-}, 6^{+}\right), \\
& A_{6}^{F+}\left(1^{-}, 2^{-}, 3^{-}, 4^{-}, 5^{-}, 6^{+}\right), \\
& A_{6}^{F+}\left(1^{-}, 2^{-}, 3^{-}, 4^{-}, 5^{-}, 6^{-}\right) .
\end{aligned}
$$

In addition, we obtained the generic NMHV (four-minus) and $\mathrm{N}^{2} \mathrm{MHV}$ (five-minus) amplitudes with seven or more external legs. The results are fairly lengthy. While the complexity of the expressions (3.9) for the MHV amplitudes from $F^{3}$ is at the same level as that of the Parke-Taylor amplitudes (3.8), the forms we find in the higher NMHV sectors become quite complex in comparison with the corresponding YM amplitudes. Of course, they can still be evaluated numerically quite easily, so that one can test, for example, the required independence of the expressions from the reference vector $\xi$. We also computed the $F^{3}$ amplitudes numerically from Feynman diagrams through six external legs, using the MADGRAPH package [54-56]; the Feynman vertices for the $F^{3}$ interaction were generated by FeynRules [57]. We found complete numerical agreement with the CSW expressions. For the six-point amplitudes with three positive and three negative helicities, the agreement is with the sum of the self-dual and anti-self-dual contributions, $A_{6}^{F+}+A_{6}^{F-}$ (see figure 2). 
One NMHV amplitude that can be simplified easily from the CSW representation is the five-point case,

$$
A_{5}^{F+}\left(1^{-}, 2^{-}, 3^{-}, 4^{-}, 5^{+}\right)=i\left[\frac{\langle 12\rangle^{2}[25]^{2}[15]}{[12][23][34][45]}+\frac{\langle 34\rangle^{2}[35]^{2}[45]}{[12][23][34][15]}+2 \frac{\langle 14\rangle^{2}\langle 13\rangle\langle 24\rangle}{[23]\langle 45\rangle\langle 15\rangle}\right] \text {. }
$$

The $\mathrm{N}^{2} \mathrm{MHV}$ all-minus five-point amplitude is also simple, in part because it is cyclicly symmetric,

$$
A_{5}^{F+}\left(1^{-}, 2^{-}, 3^{-}, 4^{-}, 5^{-}\right)=i \frac{\sum_{i=1}^{5}\left[s_{i, i+1} s_{i+1, i+2}\left(s_{i, i+2}-2 s_{i+3, i+4}\right)+\varepsilon(1,2,3,4) s_{i, i+1}\right]}{[12][23][34][45][51]}
$$

where $\varepsilon(i, j, l, m) \equiv 4 i \varepsilon_{\mu \nu \sigma \rho} k_{i}^{\mu} k_{j}^{\nu} k_{l}^{\sigma} k_{m}^{\rho}$ is the contracted Levi-Civita tensor.

We also provide one NMHV example at the six-point level,

$$
A_{6}^{F+}\left(1^{-}, 2^{-}, 3^{+}, 4^{-}, 5^{-}, 6^{+}\right)=i\left[X_{6}+\left.X_{6}\right|_{1 \leftrightarrow 5,2 \leftrightarrow 4}\right],
$$

where

$$
\begin{aligned}
X_{6}= & \frac{[36]^{3}\langle 14\rangle^{2}\langle 15\rangle^{2}\langle 45\rangle}{[23]\langle 56\rangle\langle 61\rangle[26]\left\langle 1^{-}|(2+3)| 6^{-}\right\rangle\left\langle 4^{-}|(2+3)| 6^{-}\right\rangle} \\
& +\frac{\langle 12\rangle\left\langle 1^{-}|(4+5)(4+6)| 5^{+}\right\rangle}{\langle 23\rangle[45][46] s_{56}\langle 61\rangle s_{123}\left\langle 1^{-}|(2+3)| 6^{-}\right\rangle\left\langle 3^{-}|(1+2)| 6^{-}\right\rangle} \\
& \times\left[(\langle 12\rangle[64]\langle 45\rangle[56])^{2}+\left(\left\langle 1^{-}|(4+5)| 6^{-}\right\rangle\left\langle 2^{-}|(4+5)| 6^{-}\right\rangle\right)^{2}\right] \\
& +\frac{1}{2} \frac{\langle 15\rangle^{2}\langle 24\rangle^{2}\left[(\langle 24\rangle[61]\langle 15\rangle[56])^{2}+\left(\left\langle 2^{-}|(3+4)| 6^{-}\right\rangle\left\langle 4^{-}|(2+3)| 6^{-}\right\rangle\right)^{2}\right]}{\langle 23\rangle\langle 34\rangle s_{56} s_{61} s_{234}\left\langle 2^{-}|(3+4)| 6^{-}\right\rangle\left\langle 4^{-}|(2+3)| 6^{-}\right\rangle}
\end{aligned}
$$

and $s_{i j l}=\left(k_{i}+k_{j}+k_{l}\right)^{2}$ is a multi-particle invariant.

Finally, the $\mathrm{N}^{3} \mathrm{MHV}$ all-minus six-point amplitude is cyclicly symmetric and lacks multi-particle poles, allowing us to simplify it as well,

$$
A_{6}^{F+}\left(1^{-}, 2^{-}, 3^{-}, 4^{-}, 5^{-}, 6^{-}\right)=i \frac{\sum_{i=1}^{6} f_{i}^{6-}}{[12][23][34][45][56][61]},
$$

where

$$
\begin{aligned}
f_{i}^{6-}= & s_{i, i+1}^{2}\left(s_{i-1, i}+s_{i+1, i+2}-s_{i+3, i+4}\right)+\frac{1}{2} s_{i, i+1, i+2}\left[s_{i-2, i-1, i} s_{i-1, i, i+1}+s_{i-1, i, i+1} s_{i, i+1, i+2}\right. \\
& +s_{i, i+1, i+2} s_{i-2, i-1, i}+2 s_{i+1, i+2}\left(s_{i-2, i-1, i}+s_{i, i+1}\right) \\
& \left.-s_{i, i+1} s_{i+3, i+4}-s_{i+1, i+2} s_{i+4, i+5}-3 s_{i+2, i+3} s_{i-1, i}\right] \\
& -\frac{1}{2} \varepsilon(i, i+1, i+2, i+3)\left[s_{i-2, i-1, i}+s_{i-1, i, i+1}+s_{i, i+1, i+2}+2 s_{i+1, i+2}\right] .
\end{aligned}
$$

In view of the similarity between the two sets of MHV amplitudes, YM theory and $F^{3}$, it would be very interesting to search for alternative, manifestly $\xi$-independent, allmultiplicity forms of the $F^{3}$ amplitudes, analogous to those provided by the superconformal $R$ invariants in YM theory [58]. In addition, one could imagine that - although the theory is not supersymmetrizable - there might be a compact representation in terms of momentum twistors, similar to the one for pure YM theory [59]. 


\subsection{Color-kinematics duality for $F^{3}$ amplitudes}

We now consider color-kinematics duality for $F^{3}$ amplitudes with an arbitrary number of legs. Just as in the YM case [2], we generalize the equations from the four-point example in subsection 2.1. A color-kinematic dual description of an amplitude exists, if one can find a set of kinematic numerators $n_{g}$ that satisfies three sets of equations:

- automorphism equations ensuring the total antisymmetry of the kinematic numerators at each vertex, as dictated by color, i.e. the antisymmetry of the structure constants (generalizations of eq. (2.10)),

- Jacobi equations ensuring that any numerators for diagrams whose color structures are related by Jacobi identities are related in the same way, via eq. (2.11),

- amplitude expansion - the color-ordered amplitudes are dictated by the $n_{g}$, after expanding the color factors out into traces in the fundamental representation using eq. (2.2).

Fortunately, all of the above equations are linear, which makes it straightforward to obtain solutions analytically and numerically. However, given the complexity of the expressions obtained for $\mathrm{N}^{k} \mathrm{MHV}$ amplitudes in the last subsection, it is often more convenient to test numerically whether the set of all equations allows for a solution at all. As discussed at the end of subsection 2.1, it should be equivalent (and is often simpler) to test directly the linear BCJ relations between color-ordered amplitudes [2]. For example, at the five- and six-point levels we test for the vanishing of

$$
\begin{aligned}
& s_{12} s_{45} A_{5}^{F+}(1,2,3,4,5)+s_{13} s_{24} A_{5}^{F+}(1,3,4,2,5)-s_{14}\left(s_{24}+s_{25}\right) A_{5}^{F+}(1,4,3,2,5), \\
& \left(s_{14}+s_{45}+s_{46}\right) A_{6}^{F+}(1,2,3,4,5,6)+\left(s_{14}+s_{46}\right) A_{6}^{F+}(1,2,3,5,4,6) \\
& \quad+s_{14} A_{6}^{F+}(1,2,3,5,6,4)-s_{24} A_{6}^{F+}(1,2,4,3,5,6),
\end{aligned}
$$

plus permutations of these equations.

It is amusing to note that consistency with the BCJ relations determines certain contributions that cannot be determined using factorization limits with real momentum. For example, in eq. (3.17) the term proportional to $\varepsilon(1,2,3,4)$ vanishes in all (real) collinear limits. Its coefficient is fixed, however, by imposing the vanishing of eq. (3.22).

\subsection{Recycling of numerators}

The close similarity between eqs. (3.8) and (3.9) was one motivation to explore the consistency of $F^{3}$ amplitudes with color-kinematics duality. Indeed, as will be pointed out below, at the MHV level the duality for $F^{3}$ amplitudes is already manifest, given a dual representation for YM theory.

The MHV amplitudes (3.8) in pure YM theory can be split into two pieces. The statedependent part $\langle p q\rangle^{4}$, where $p, q$ are the two negative-helicity gluons, is determined by a Grassmann integral in an on-shell superspace [60]. The state-independent remnant,

$$
A_{m}^{\mathrm{MHV}, \mathrm{rem}} \equiv \frac{i}{\langle 12\rangle\langle 23\rangle \cdots\langle m 1\rangle},
$$


carries all of the dependence on the color ordering. The BCJ relations for color-ordered amplitudes relate amplitudes with the same external states but different color ordering. Therefore at the MHV level they are equivalent to relations among the remnants. We define

$$
n_{g}^{\mathrm{MHV}, \mathrm{rem}}=\frac{n_{g}^{\mathrm{YM}, p, q}}{\langle p q\rangle^{4}}
$$

as the set of BCJ numerator factors out of which the state-independent remnant is built. For example, at the four-point level, we have

$$
\frac{i}{\langle 12\rangle\langle 23\rangle\langle 34\rangle\langle 41\rangle}=\frac{n_{s}^{\mathrm{MHV}, \mathrm{rem}}}{s}+\frac{n_{t}^{\mathrm{MHV}, \mathrm{rem}}}{t} .
$$

Given the existence of duality-satisfying numerators $n_{g}^{\mathrm{YM}, p, q}$ for MHV amplitudes of arbitrary multiplicity in YM theory, we can easily obtain dual numerators for other amplitudes, provided that they allow a similar splitting, into a factor that is independent of the color ordering, multiplied by the same remnant factor as in eq. (3.24). The prime example of amplitudes obeying this criterion are the MHV amplitudes from the $F_{\mathrm{SD}}^{3}$ sector. They split according to

$$
A_{m}^{F_{+}}\left(\ldots, j^{-}, \ldots, k^{-}, \ldots, l^{-}, \ldots\right)=\langle j k\rangle^{2}\langle k l\rangle^{2}\langle l j\rangle^{2} \times \frac{i}{\langle 12\rangle\langle 23\rangle \cdots\langle m 1\rangle} .
$$

Thus the numerator for an $F^{3} \mathrm{MHV}$ amplitude can be obtained trivially from the numerator for a pure YM MHV amplitude via

$$
n_{g}^{F^{+}, j, k, l}=\langle j k\rangle^{2}\langle k l\rangle^{2}\langle l j\rangle^{2} n_{g}^{\mathrm{MHV}, \mathrm{rem}}=\frac{\langle j k\rangle^{2}\langle k l\rangle^{2}\langle l j\rangle^{2}}{\langle p q\rangle^{4}} n_{g}^{\mathrm{YM}, p, q},
$$

where $j, k, l$ and $p, q$ denote the negative-helicity gluons in the $F^{+}$and YM amplitudes respectively.

Another example is provided by the all-minus four-point $F^{3}$ amplitude (3.10). Here we use the splitting,

$$
A_{4}^{F^{+}}\left(1^{-}, 2^{-}, 3^{-}, 4^{-}\right)=-2 s t u \times \frac{i}{[12][23][34][41]},
$$

where the remnant can be identified as the parity conjugate of the four-point MHV remnant in eq. (3.24). This splitting works because stu is symmetric under all permutations, and hence independent of the color ordering. Therefore a solution can be obtained from a solution for a pure YM four-point amplitude via

$$
n_{g}^{F^{+},(----)}=-2 s t u\left[n_{g}^{\mathrm{MHV}, \mathrm{rem}}\right]^{\dagger}=-2 \frac{s t u}{[p q]^{4}}\left[n_{g}^{\mathrm{YM}, p, q}\right]^{\dagger},
$$

where the dagger denotes the spinor conjugation operation (parity).

Of course, one could have arrived at the same result in the conventional way described in the last subsection. For the four-point amplitude, the cubic tree graphs are universal 
and thus all contributions to $A_{4}^{F^{+}}\left(1^{-}, 2^{-}, 3^{-}, 4^{-}\right)$and permutations are shown in figure 1. While the automorphism equations (2.5) and the Jacobi identities (2.12) thus agree with pure YM theory, the amplitude equations are now supposed to yield the appropriate $F^{3}$ amplitudes, for example,

$$
A_{4}^{F+}\left(1^{-}, 2^{-}, 3^{-}, 4^{-}\right)=\frac{n_{s}}{s}+\frac{n_{t}}{t} .
$$

The resulting set of equations determining the numerators does not have a unique solution. Instead, it exhibits the same one-parameter generalized gauge freedom as explored for pure YM theory in ref. [2].

This observation does not come as a surprise; it follows from the existence of the recycled numerators (3.30) for this particular amplitude. The YM remnant numerators possess the generalized gauge freedom, and this property carries over to the recycled numerator via eq. (3.30).

A particularly nice numerator solution, reproducing eq. (3.10), reads

$$
\begin{aligned}
n_{s} & =-\frac{2 i}{3} \frac{s^{2} t(u-t)}{[12][23][34][41]}, \\
n_{t} & =-\frac{2 i}{3} \frac{s t^{2}(u-s)}{[23][34][41][12]}, \\
n_{u} & =-\frac{2 i}{3} \frac{t u^{2}(s-t)}{[14][42][23][31]},
\end{aligned}
$$

where the generalized gauge freedom has been used to find a particularly symmetric expression. In fact, this is one of the situations where one can find a so-called symmetric numerator, that is, a function assigning every graph with a particular labeling a valid BCJ numerator. The following expression reproduces eq. (3.32) for $n_{s}=n[1,2,3,4], n_{t}=n[2,3,4,1]$ and $n_{u}=[3,1,4,2]$ :

$$
n[a, b, c, d]=-\frac{2 i}{3} \frac{s_{a b} s_{b c}}{[a b][b c][c d][d a]} s_{a b}\left(s_{a c}-s_{b c}\right) .
$$

Symmetric numerators for five- and six-point MHV YM amplitudes have been identified and discussed in ref. [61].

\subsection{Monodromy relations and $F^{3}$ amplitudes}

At the end of subsection 2.1 we discussed the likely equivalence of the BCJ amplitude relations and the existence of color-kinematic dual representations. Assuming this equivalence, there is a very good argument in favor of the existence of color-kinematic dual representations for all $F^{3}$ amplitudes, which originates in the string-theory monodromy relations [3-5]. These relations connect open-string amplitudes with different cyclic orderings of the external legs. They can be derived by deforming the contours for the integration of open-string vertex operators along the boundary of the world-sheet [62].

The monodromy relations read,

$$
\begin{aligned}
& A^{\text {string }}(1,2,3,4, \ldots, m)+e^{i \alpha^{\prime} \pi s_{12}} A^{\text {string }}(2,1,3,4, \ldots, m) \\
& \quad+e^{i \alpha^{\prime} \pi\left(s_{12}+s_{13}\right)} A^{\text {string }}(2,3,1,4, \ldots, m) \\
& \quad+\cdots+e^{i \alpha^{\prime} \pi\left(s_{12}+s_{13}+\ldots+s_{1, m-1}\right)} A^{\text {string }}(2,3,4, \ldots, m-1,1, m)=0 .
\end{aligned}
$$


We now expand the exponential in eq. (3.34) in $\alpha^{\prime}$, and also the string amplitude,

$$
A^{\text {string }}=A^{\mathrm{YM}}+\alpha^{\prime} A^{\alpha^{\prime}}+\cdots,
$$

where the first correction $A^{\alpha^{\prime}}$ is directly proportional to the $F^{3}$ amplitude, $A^{\alpha^{\prime}} \propto A^{F}$. Different terms in the expansion of eq. (3.34) provide different information. By choosing the polarization vectors to be real (linear polarizations), we can consider $A^{\mathrm{YM}}$ and $A^{\alpha^{\prime}}$ to be real. (Later we can take linear combinations with complex coefficients to obtain helicity amplitudes.)

The $\mathcal{O}\left(\alpha^{\prime 0}\right)$ term in the expansion leads to the standard photon $(\mathrm{U}(1))$ decoupling identity for YM amplitudes,

$$
\begin{aligned}
0= & A^{\mathrm{YM}}(1,2,3,4, \ldots, m)+A^{\mathrm{YM}}(2,1,3,4, \ldots, m) \\
& +A^{\mathrm{YM}}(2,3,1,4, \ldots, m)+\cdots+A^{\mathrm{YM}}(2,3,4, \ldots, m-1,1, m),
\end{aligned}
$$

while the imaginary part of the $\mathcal{O}\left(\alpha^{\prime}\right)$ term yields the BCJ relations for YM amplitudes,

$$
\begin{aligned}
0= & s_{12} A^{\mathrm{YM}}(2,1,3, \ldots, m)+\left(s_{12}+s_{13}\right) A^{\mathrm{YM}}(2,3,1,4, \ldots, m) \\
& +\cdots+\left(s_{12}+s_{13}+\ldots+s_{1, m-1}\right) A^{\mathrm{YM}}(2,3,4, \ldots, m-1,1, m) .
\end{aligned}
$$

In contrast, the real part of the $\mathcal{O}\left(\alpha^{\prime}\right)$ term and the imaginary part of the $\mathcal{O}\left(\alpha^{\prime 2}\right)$ term yield, respectively, the photon-decoupling and BCJ relations for the $F^{3}$ amplitudes,

$$
\begin{aligned}
\mathcal{O}\left(\alpha^{1}\right): 0= & A^{\alpha^{\prime}}(1,2,3,4, \ldots, m)+A^{\alpha^{\prime}}(2,1,3,4, \ldots, m) \\
& +A^{\alpha^{\prime}}(2,3,1,4, \ldots, m)+\cdots+A^{\alpha^{\prime}}(2,3,4, \ldots, m-1,1, m) \\
\mathcal{O}\left(\alpha^{\prime 2}\right): \quad 0= & s_{12} A^{\alpha^{\prime}}(2,1,3, \ldots, m)+\left(s_{12}+s_{13}\right) A^{\alpha^{\prime}}(2,3,1,4, \ldots, m) \\
& +\cdots+\left(s_{12}+s_{13}+\ldots+s_{1, m-1}\right) A^{\alpha^{\prime}}(2,3,4, \ldots, m-1,1, m) .
\end{aligned}
$$

In other words, the BCJ relations for the $F^{3}$ amplitudes can be derived simply by using the string-theory monodromy relations, and the fact that $F^{3}$ is the unique operator appearing at $\mathcal{O}\left(\alpha^{\prime}\right)$ in the low-energy effective action of the open bosonic string.

We have tested the $F^{3}$ amplitudes for the availability of color-kinematic dual numerators (satisfying antisymmetry (2.10) and kinematic Jacobi relations (2.11)). We have found such a representation to be indeed present in all cases. At the four-point level, the representations can be exhibited analytically; the only independent cases are the all-minus and MHV cases discussed above. For the five- and six-point cases we generally relied on numerical checks.

Interestingly, the representations for $m$-point amplitudes exhibit the same generalized gauge freedom as the numerators for YM amplitudes with the same number of external legs. The dimension of the vector space of numerator solutions parametrizing the generalized gauge freedom in pure gauge theory is

$$
\operatorname{dim}_{\text {ggf }}=(m-2) !-(m-3) !,
$$

which is exactly the dimension of the solution space we find for color-kinematic dual representations of $F^{3}$ amplitudes. 
Given the availability of the dual representations for amplitudes produced by the $F^{3}$ modification of pure gauge theory, we expect to be able to use the double-copy construction to generate amplitudes in a theory of gravity. After carrying out this procedure in the next section, we then address the question: to which theory of gravity do these amplitudes belong?

\section{Squaring to gravity}

In the last section we established the BCJ relations and color-kinematic dual representations for gauge-theory amplitudes with one insertion of a vertex originating from the $F^{3}$ operator. In YM theory, the existence of a dual representation is a sufficient criterion for the double-copy construction of amplitudes in Einstein gravity and its various supersymmetrizations, given that the amplitudes behave well for large complex BCFW shifts [9]. Here we consider the set of gravitational amplitudes obtained by applying the double-copy construction to the $F^{3}$-deformed gauge-theory amplitudes from the last section. The amplitudes are not as well behaved under large shifts as in the YM case. On the other hand, we know there is a gravity-gauge-theory relation of some type, because the $F^{3}$ operator is part of the effective action for the open bosonic string. We will compare the double-copy results with those derived from the KLT relations and discuss the modified gravitational action from which they can be obtained.

\subsection{Set of amplitudes}

In order to obtain the double copy of the $F^{3}$ amplitudes, we insert two copies of the numerators calculated in the last section into eq. (2.17). Let us first consider the class of amplitudes resulting from combining numerators from two $F^{+}$-amplitudes, which will be denoted ${ }^{2}$ by a superscript $R^{+}$. Unless otherwise noted, we will always combine pairs of external gluons $g^{ \pm}$with the same helicity in the YM amplitudes, so as to yield only gravitons $G^{ \pm}$after double-copying:

$$
G^{+}=g^{+} \otimes g^{+} \quad \text { and } \quad G^{-}=g^{-} \otimes g^{-} .
$$

Choosing opposite helicities, $g^{ \pm} \otimes g^{\mp}$, will generate the dilaton and axion scalar states, as we will see later.

The basic building blocks for the set of gravitational amplitudes are the three-point identical-helicity vertices, which are exactly the square of their $F^{3}$ counterparts:

$$
M_{3}^{R+}\left(1^{-}, 2^{-}, 3^{-}\right)=-i(\langle 12\rangle\langle 23\rangle\langle 31\rangle)^{2}=i\left[A_{3}^{F+}\left(1^{-}, 2^{-}, 3^{-}\right)\right]^{2} .
$$

Using the notation introduced in eq. (2.16), we can write eq. (4.2) as

$$
M_{3}^{R+}\left(1^{-}, 2^{-}, 3^{-}\right)=\operatorname{KLT}_{\mathrm{FT}, 3}\left[A_{3}^{F^{+}}\left(1^{-}, 2^{-}, 3^{-}\right), A_{3}^{F^{+}}\left(1^{-}, 2^{-}, 3^{-}\right)\right] .
$$

\footnotetext{
${ }^{2}$ In contrast to the + in $F^{+}$introduced in section 3, the superscript + in $R^{+}$does not imply that the resulting amplitudes are matrix elements of the self-dual part of some operator. We will see below that this is not the case.
} 
For the four-graviton amplitude with three negative-helicity gravitons, the double-copy construction yields

$$
\begin{aligned}
M_{4}^{R+}\left(1^{-}, 2^{-}, 3^{-}, 4^{+}\right) & =\frac{(\langle 12\rangle\langle 23\rangle\langle 31\rangle)^{4}}{\langle 12\rangle^{8}} M_{4}\left(1^{-}, 2^{-}, 3^{+}, 4^{+}\right) \\
& =-i([41]\langle 13\rangle[34])^{2} \frac{\langle 12\rangle\langle 23\rangle\langle 31\rangle}{[12][23][31]}
\end{aligned}
$$

which is in agreement with

$$
\mathrm{KLT}_{\mathrm{FT}, 4}\left[A_{4}^{F^{+}}\left(1^{-}, 2^{-}, 3^{-}, 4^{+}\right), A_{4}^{F^{+}}\left(1^{-}, 2^{-}, 3^{-}, 4^{+}\right)\right] .
$$

In fact, the general form of the $m$-graviton $R^{+}$amplitudes with exactly three negativehelicity gravitons,

$$
\begin{aligned}
M_{m}^{R+}\left(1^{+}\right. & \left., \ldots, j^{-}, \ldots, k^{-}, \ldots, l^{-}, \ldots, m^{+}\right) \\
& =\frac{(\langle j k\rangle\langle k l\rangle\langle l j\rangle)^{4}}{\langle p q\rangle^{8}} M_{m}\left(1^{+}, \ldots, p^{-}, \ldots, q^{-}, \ldots, m^{+}\right)
\end{aligned}
$$

follows straightforwardly from eq. (3.28).

Consider now the first amplitude with four negative-helicity gravitons. One finds that the double-copy construction yields

$$
\begin{aligned}
M_{4}^{R+}\left(1^{-}, 2^{-}, 3^{-}, 4^{-}\right) & =4 \frac{(s t u)^{2}}{[34]^{8}} M_{4}\left(1^{-}, 2^{-}, 3^{+}, 4^{+}\right) \\
& =4 i \text { stu } \frac{\langle 12\rangle\langle 23\rangle\langle 34\rangle\langle 41\rangle}{[12][23][34][41]}
\end{aligned}
$$

For higher multiplicities, and excluding the amplitudes with three negative-helicity gravitons, the analytic expressions get unwieldy. Nevertheless, double copies can be obtained easily once a (possibly numerically determined) set of numerators for the $F^{3}$ amplitudes is available. Using the results from the previous section, we have computed the following gravitational amplitudes:

$$
\begin{aligned}
& M_{5}^{R+}\left(1^{-}, 2^{-}, 3^{-}, 4^{-}, 5^{+}\right), \\
& M_{5}^{R+}\left(1^{-}, 2^{-}, 3^{-}, 4^{-}, 5^{-}\right), \\
& M_{6}^{R+}\left(1^{-}, 2^{-}, 3^{-}, 4^{-}, 5^{+}, 6^{+}\right), \\
& M_{6}^{R+}\left(1^{-}, 2^{-}, 3^{-}, 4^{-}, 5^{-}, 6^{+}\right), \\
& M_{6}^{R+}\left(1^{-}, 2^{-}, 3^{-}, 4^{-}, 5^{-}, 6^{-}\right) .
\end{aligned}
$$

\subsection{Consistency checks}

With the set of amplitudes (4.7) and (4.10) at one's disposal, one can perform various consistency checks. An immediate check is the total symmetry under exchange of any pair of equal-helicity gravitons. This symmetry is not manifest in the double-copy construction, but we verified it for all amplitudes, either analytically or numerically. 
Another set of relations to be satisfied by the gravitational amplitudes can be derived from collinear and soft factorization. For example, suppose the external momenta $k_{4}$ and $k_{5}$ are collinear, obeying $k_{4} \approx z k_{P}$ and $k_{5} \approx(1-z) k_{P}$ with $k_{P}=k_{4}+k_{5}$ and $z \in[0,1]$. Then the following relation has to hold:

$$
\begin{aligned}
M_{6}^{R+}\left(1^{-}, 2^{-}, 3^{-}, 4^{-}, 5^{+}, 6^{+}\right) \stackrel{k_{4} \| k_{5}}{\longrightarrow} & \operatorname{Split}_{-}\left(4^{-}, 5^{+}\right) M_{5}^{R+}\left(1^{-}, 2^{-}, 3^{-}, P^{+}, 6^{+}\right) \\
& +\operatorname{Split}_{+}\left(4^{-}, 5^{+}\right) M_{5}^{R+}\left(1^{-}, 2^{-}, 3^{-}, P^{-}, 6^{+}\right) .
\end{aligned}
$$

The gravitational splitting amplitudes applicable to the above situation are [46]

$$
\begin{aligned}
& \operatorname{Split}_{-}\left(4^{-}, 5^{+}\right)=-\frac{(1-z)^{3}}{z} \frac{\langle 45\rangle}{[45]}, \\
& \operatorname{Split}_{+}\left(4^{-}, 5^{+}\right)=-\frac{z^{3}}{1-z} \frac{[45]}{\langle 45\rangle} .
\end{aligned}
$$

In other factorization channels one also needs

$$
\begin{aligned}
& \operatorname{Split}_{+}\left(4^{-}, 5^{-}\right)=-\frac{1}{z(1-z)} \frac{\langle 45\rangle}{[45]}, \\
& \text { Split_}_{-}\left(4^{-}, 5^{-}\right)=0
\end{aligned}
$$

plus the complex conjugate splitting amplitudes. (See ref. [46], section 5, for subtleties related to testing the collinear limits of gravity amplitudes.) The real collinear limits of the ' $R^{+}$' amplitudes have exactly the same form as in Einstein gravity, because the threepoint amplitudes in this theory, eq. (4.2), vanish in the real collinear limit. Hence the collinear singularities come just from the Einstein triple-graviton vertex.

Similarly, the soft limits will involve the standard soft-graviton factor for Einstein gravity. An example of a soft-limit relation is

$$
M_{5}^{R+}\left(1^{-}, 2^{-}, 3^{-}, 4^{-}, 5^{+}\right) \stackrel{k_{4} \rightarrow 0}{\longrightarrow} \operatorname{Soft}\left(4^{-}\right) M_{4}^{R+}\left(1^{-}, 2^{-}, 3^{-}, 5^{+}\right),
$$

where the soft factor in the above equation is given by

$$
\operatorname{Soft}\left(4^{-}\right)=\frac{\langle 14\rangle[13][24][51]+\langle 24\rangle[14][23][52]}{[14][24][34][54]}
$$

and a general form can be found in ref. [46].

We tested all possible collinear and soft limits for the amplitudes in eq. (4.10). In addition, we tested that the collinear and soft limits of our formula (4.7) for amplitudes with three negative-helicity gravitons are consistent up to the ten-point level. In short, the set of amplitudes obtained by double-copying the $F^{3}$ amplitudes satisfies all expected collinear and soft limits for multi-graviton amplitudes.

While the agreement between the result of the double-copy (DC) construction and the application of the field-theory KLT relations $\mathrm{KLT}_{\mathrm{FT}, m}$ was already noted in eqs. (4.3) and (4.6) for three- and four-point cases, we have compared the results of the two methods numerically for all other available amplitudes through six points. For all amplitudes that we tested, we find

$$
\mathrm{DC}\left[A^{F^{+}}, A^{F^{+}}\right]=\operatorname{KLT}_{\mathrm{FT}}\left[A^{F^{+}}, A^{F^{+}}\right] .
$$


Thus for these cases the double-copy construction is equivalent to applying the field-theory limit of the KLT relations to the $F^{3}$ amplitudes. By parity we also have, for the conjugate helicity configuration,

$$
\mathrm{DC}\left[A^{F^{-}}, A^{F^{-}}\right]=\operatorname{KLT}_{\mathrm{FT}}\left[A^{F^{-}}, A^{F^{-}}\right] .
$$

In one of the cases that we tested, the two towers in figure 2 overlap, and an $F^{3}$ amplitude can receive nonzero contributions from both $A^{F^{+}}$and $A^{F^{-}}$. This helicity configuration, in the six-graviton case, is $(---+++)$. In this case it is also of interest to test for the off-diagonal double-copy or KLT construction. We find that in this case both constructions vanish:

$$
\mathrm{DC}\left[A^{F^{+}}, A^{F^{-}}\right]=\operatorname{KLT}_{\mathrm{FT}}\left[A^{F^{+}}, A^{F^{-}}\right]=0,
$$

for $(---+++)$. It would be interesting to investigate whether this is true more generally.

\subsection{KLT relations and effective actions for the bosonic string}

Next we address the question as to which gravitational action produces the ' $R^{+}$' amplitudes from the previous subsection. The fact that the double-copy construction yields results equivalent to those obtained by employing the field-theory KLT relations clearly suggests that the amplitudes may originate from corrections to the Einstein-Hilbert action that appear in the low-energy effective action of the closed bosonic string.

The operator $F^{3}$ appears at $\mathcal{O}\left(\alpha^{\prime}\right)$ in the low-energy effective action of the open bosonic string. Thus, the resulting gravitational amplitudes from double-copying an amplitude with one insertion of $F^{3}$ should appear at $\mathcal{O}\left(\alpha^{\prime 2}\right)$ in a gravitational effective action. The low-energy effective action for the closed bosonic string reads [63],

$$
\begin{aligned}
S=-\frac{2}{\kappa^{2}} \int \mathrm{d}^{4} x \sqrt{g}[ & R-2\left(\partial_{\mu} \phi\right)^{2}-\frac{1}{12} H^{2} \\
& +\alpha^{\prime} \frac{1}{4} e^{-2 \phi} G_{2} \\
& \left.+\alpha^{\prime 2} e^{-4 \phi}\left(\frac{1}{48} I_{1}+\frac{1}{24} G_{3}\right)+\mathcal{O}\left(\alpha^{\prime 3}\right)\right],
\end{aligned}
$$

where the variable $\phi$ denotes the dilaton and $H=d B$ is the outer derivative of the totally antisymmetric tensor $B_{\mu \nu}$. In addition, $G_{2}=R_{\mu \nu \rho \sigma} R^{\mu \nu \rho \sigma}-4 R_{\mu \nu} R^{\mu \nu}+R^{2}$ is the usual topological Gauss-Bonnet contribution with two powers of the Riemann tensor. The two terms appearing at $\mathcal{O}\left(\alpha^{\prime 2}\right)$ are defined by

$$
I_{1}=R_{\alpha \beta}^{\mu \nu} R_{\sigma \rho}^{\alpha \beta} R_{\mu \nu}^{\sigma \rho} \quad \text { and } \quad G_{3}=I_{1}-2 R_{\alpha \beta}^{\mu \nu} R_{\beta \gamma}^{\nu \sigma} R_{\gamma \alpha}^{\sigma \mu} .
$$

Both of the $\mathcal{O}\left(\alpha^{\prime 2}\right)$ terms in the above equation, $I_{1}$ and $G_{3}$, could in principle result in corrections at $\mathcal{O}\left(\alpha^{\prime 2}\right)$. However, expanding the terms around flat Minkowski space, one can show [63] that only the first term, $I_{1}$, contributes to $\mathcal{O}\left(\alpha^{\prime 2}\right)$ terms in the four-graviton amplitudes in which we are interested (although the $G_{3}$ term does contribute to mixed dilaton-graviton four-point amplitudes).

Below, we will refer to the particular combination of operators at $\mathcal{O}\left(\alpha^{\prime 2}\right)$ in the eq. (4.19) simply as $R^{3}$. As we will show below, the vertices originating from the operators appearing at $\mathcal{O}\left(\alpha^{\prime 2}\right)$ are insufficient to yield the set of amplitudes obtained from 
double-copying the amplitudes linear in $F^{3}$. One needs, in addition, to consider contributions from two insertions of the vertices appearing at $\mathcal{O}\left(\alpha^{\prime}\right)$ in eq. (4.19), which are generated by the operator $e^{-2 \phi} G_{2}$.

An immediate obstacle for a direct comparison between the double-copy results and amplitudes resulting from the action (4.19) is the availability of amplitudes resulting from an insertion of the operator $R^{3}$. While the implementation of CSW-like rules for amplitudes in the $F^{3}$ sector above was straightforward (see section 3), the situation in gravity is more subtle: an attempt to generalize the CSW-formalism to gravity resulted in complicated rules requiring BCFW-like shifts [64] which renders calculations cumbersome. As an alternative, one can use all-line BCFW-shifts $[65,66]$. However, this method is limited to particular helicity amplitudes that vanish as the complex shifted momentum becomes large; it cannot be applied to all helicity configurations.

Let us start with the available amplitudes. At the three-point level, the operator $R^{3}$ generates nonzero amplitudes for the three-graviton helicity configurations $(---)$ and $(+++)$, while the configurations $(\mp \mp \pm)$ receive no contribution from it. For convenience, we will normalize the operator $R^{3}$ so that its three-graviton amplitudes are given by $M_{3}^{R+}$ in eq. (4.2). At the level of four gravitons, Cohen, Elvang and Kiermaier [30] calculated the all-minus amplitude for pure gravity with one insertion of a vertex originating in $R^{3}$. An anti-holomorphic all-line shift leads to only one contributing BCFW diagram, with two cubic vertices and a graviton exchange, and then a sum over all possible permutations of the external states, $\mathcal{P}(1234)$, yielding ${ }^{3}$

$$
M_{4}^{R^{3}, \text { all-line shift }}\left(1^{-}, 2^{-}, 3^{-}, 4^{-}\right)=\frac{i}{4} \sum_{\mathcal{P}(1234)} \frac{\langle 12\rangle^{5}\langle 34\rangle^{2}}{[12]} \frac{[1 \xi]^{2}[2 \xi]^{2}}{[3 \xi]^{2}[4 \xi]^{2}} .
$$

We can simplify this expression to

$$
\begin{aligned}
M_{4}^{R^{3}, \text { all-line shift }}\left(1^{-}, 2^{-}, 3^{-}, 4^{-}\right) & =10 i \operatorname{stu} \frac{\langle 12\rangle\langle 23\rangle\langle 34\rangle\langle 41\rangle}{[12][23][34][41]} \\
& =\frac{5}{2} M_{4}^{R+}\left(1^{-}, 2^{-}, 3^{-}, 4^{-}\right),
\end{aligned}
$$

where $M_{4}^{R+}$ is the four-graviton amplitude constructed from either the double-copy formula or the KLT relations, and given in eq. (4.9).

The proportionality of the $R^{3}$ amplitude to the result from applying field-theory KLT relations to the all-minus four-point $F^{3}$ amplitudes was already noted in ref. [30]. However, the two expressions are not equal; there is an prefactor of $\frac{5}{2}$ in front of $M_{4}^{R+}$ which needs to be explained. The explanation is that the double-copy construction (or equivalently the field-theory KLT relations) includes diagrams with axion and dilaton exchange, not just the pure-graviton contributions in the computation $(4.21) .{ }^{4}$

The real scalar dilaton $\phi$ and pseudoscalar axion $a$ are given in terms of a complex scalar field $\varphi$ and its complex conjugate $\bar{\varphi}$ as (following the conventions of ref. [67])

$$
\phi=\frac{1}{2}(\varphi+\bar{\varphi}) \quad \text { and } \quad a=\frac{i}{2}(\varphi-\bar{\varphi}),
$$

${ }^{3}$ Compared to the result in ref. [30], our conventions lead to an additional prefactor of $i$ in eq. (4.21).

${ }^{4}$ We thank Tim Cohen, Henriette Elvang and Michael Kiermaier for this explanation. 
respectively. Amplitudes involving $\varphi$ and $\bar{\varphi}$ can be obtained via the double-copy construction or KLT relations by combining amplitude pairs whose corresponding gluons have opposite helicity,

$$
\varphi=g^{-} \otimes g^{+} \quad \text { and } \quad \bar{\varphi}=g^{+} \otimes g^{-} .
$$

At $\mathcal{O}\left(\alpha^{\prime 2}\right)$, there are contributions to the amplitude with four negative-helicity gravitons from exchanges of a dilaton or axion between two three-point vertices, each generated by an insertion of the operator $e^{-2 \phi} G_{2}$ in eq. (4.19).

The necessary three-point vertices, with two gravitons and either $\varphi$ or $\bar{\varphi}$, are easy to work out by expanding the operator $e^{-2 \phi} G_{2}$. They are simplest to normalize (relative to the $R^{3}$ amplitude) by using the KLT relations. The results are

$$
\begin{aligned}
M_{3}\left(1^{-}, 2^{-}, 3^{\varphi}\right)=M_{3}\left(1^{-}, 2^{-}, 3^{\bar{\varphi}}\right) & =\operatorname{KLT}_{\mathrm{FT}, 3}\left[A_{3}^{F+}\left(1^{-}, 2^{-}, 3^{\mp}\right), A_{3}\left(1^{-}, 2^{-}, 3^{ \pm}\right)\right] \\
& =-i\langle 12\rangle^{4}
\end{aligned}
$$

Angular-momentum conservation implies a vanishing result for the case of opposite helicity gravitons:

$$
M_{3}\left(1^{-}, 2^{+}, 3^{\varphi}\right)=M_{3}\left(1^{-}, 2^{+}, 3^{\bar{\varphi}}\right)=0 .
$$

The results (4.26) and (4.27) are also valid when the scalar particle is off-shell. We remark that these three-point vertices all vanish in the limit of real collinear kinematics. Therefore the collinear limits we studied in the previous subsection can receive no contributions from terms generated by two insertions of $e^{-2 \phi} G_{2}$.

Next we compute the scalar exchange contribution to the all-minus four-graviton amplitude. We connect two vertices of the form (4.26) with a scalar propagator, include a factor of two to account for both $\varphi$ and $\bar{\varphi}$ exchange, and sum over all three exchange channels, to obtain

$$
M_{4}^{\left(\phi R^{2}\right)^{2}}\left(1^{-}, 2^{-}, 3^{-}, 4^{-}\right)=-2 i\left(\frac{\langle 12\rangle^{4}\langle 34\rangle^{4}}{s_{12}}+\frac{\langle 13\rangle^{4}\langle 24\rangle^{4}}{s_{13}}+\frac{\langle 23\rangle^{4}\langle 41\rangle^{4}}{s_{23}}\right) .
$$

(In the dilaton/axion basis (4.24), all the contributions come from the dilaton; the diagrams from axion exchange cancel.) The amplitude in eq. (4.28) turns out to be precisely $-\frac{3}{2}$ times the KLT result (4.9).

In summary, the KLT expression (4.9) can be decomposed as,

$$
\begin{aligned}
M_{4}^{R+}\left(1^{-}, 2^{-}, 3^{-}, 4^{-}\right) & =M_{4}^{R^{3}, \text { all-line shift }}\left(1^{-}, 2^{-}, 3^{-}, 4^{-}\right)+M_{4}^{\left(\phi R^{2}\right)^{2}}\left(1^{-}, 2^{-}, 3^{-}, 4^{-}\right)( \\
& =\left(\frac{5}{2}-\frac{3}{2}\right) M_{4}^{R+}\left(1^{-}, 2^{-}, 3^{-}, 4^{-}\right)
\end{aligned}
$$

This result shows that applying the double-copy construction to a pair of amplitudes, each with one insertion of a vertex originating in $F^{+}$, yields graviton amplitudes with gravitons and dilaton/axions on internal lines. Although this was the simplest possible example, it is not difficult to verify this behavior for other amplitudes, at least when the pure $R^{3}$ contribution is available. For example, we have compared the double-copy result for the 
five-point all-minus amplitude with an all-line-shift calculation of the $R^{3}$ contribution, and we find the expected result:

$$
\begin{aligned}
M_{5}^{R+}\left(1^{-}, 2^{-}, 3^{-}, 4^{-}, 5^{-}\right) & =M_{5}^{R^{3}, \text { all-line shift }}\left(1^{-}, 2^{-}, 3^{-}, 4^{-}, 5^{-}\right)+M_{5}^{\left(\phi R^{2}\right)^{2}}\left(1^{-}, 2^{-}, 3^{-}, 4^{-}, 5^{-}\right) \\
& =\left(\frac{5}{2}-\frac{3}{2}\right) M_{5}^{R+}\left(1^{-}, 2^{-}, 3^{-}, 4^{-}, 5^{-}\right)
\end{aligned}
$$

The second contribution on the right-hand side of the first equation contains a four-point subdiagram with dilaton exchange (the axion exchange contribution again vanishes). For general amplitudes, the $R^{3}$ and dilaton/axion contributions presumably need not be proportional, although they are in the two all-minus cases we computed explicitly, eqs. (4.30) and (4.31).

For the four-graviton helicity configuration $(---+)$, the vanishing of the oppositehelicity three-point amplitude $M_{3}^{\mathcal{O}\left(\alpha^{\prime}\right)}\left(1^{-}, 2^{+}, 3^{\varphi}\right)$ in eq. (4.27) implies that the KLTconstructed amplitude $M_{4}^{R+}\left(1^{-}, 2^{-}, 3^{-}, 4^{+}\right)$given in eq. (4.5) should have no contributions from dilaton/axion exchange. That is, it should come purely from $R^{3}$ :

$$
M_{4}^{R+}\left(1^{-}, 2^{-}, 3^{-}, 4^{+}\right)=M_{4}^{R^{3}}\left(1^{-}, 2^{-}, 3^{-}, 4^{+}\right) .
$$

We can test these results against a Feynman-diagram computation by van Nieuwenhuizen and $\mathrm{Wu}[68]$ of the pure-graviton $R^{3}$ amplitudes for the helicity configurations (----) and $(---+) .{ }^{5}$ In order to avoid discussing phases and operator normalization conventions, we take the magnitude of the ratio of amplitudes they computed,

$$
\left|\frac{M_{4}^{\mathrm{vNW}}\left(1^{-}, 2^{-}, 3^{-}, 4^{-}\right)}{M_{4}^{\mathrm{vNW}}\left(1^{-}, 2^{-}, 3^{-}, 4^{+}\right)}\right|=\left|\frac{\frac{15}{4} i s t u}{\frac{3}{8} i s t u}\right|=10 .
$$

Our corresponding ratio, from eqs. (4.5), (4.9) and (4.23), is,

$$
\frac{5}{2}\left|\frac{M_{4}^{R+}\left(1^{-}, 2^{-}, 3^{-}, 4^{-}\right)}{M_{4}^{R+}\left(1^{-}, 2^{-}, 3^{-}, 4^{+}\right)}\right|=\frac{5}{2} \times \frac{4 s t u}{s t u}=10,
$$

in perfect agreement with eq. (4.33).

The final four-graviton helicity configuration to consider at $\mathcal{O}\left(\alpha^{\prime 2}\right)$ is $(--++)$. There is no contribution to this amplitude from one insertion of the operator $R^{3}$ [68]. Also, in the effective action of the closed superstring there is no contribution at this order (the first puregraviton correction is at $\mathcal{O}\left(\alpha^{\prime 3}\right)$ from the $R^{4}$ operator). Finally, there is no contribution from the KLT-square of two $F^{3}$ amplitudes, because the component amplitudes vanish,

$$
M_{4}^{R+}\left(1^{-}, 2^{-}, 3^{+}, 4^{+}\right) \equiv \operatorname{KLT}_{\mathrm{FT}, 4}\left[A_{4}^{F^{+}}\left(1^{-}, 2^{-}, 3^{+}, 4^{+}\right), A_{4}^{F^{+}}\left(1^{-}, 2^{-}, 3^{+}, 4^{+}\right)\right]=0 .
$$

On the other hand, there is a nonzero contribution in the closed bosonic string, and this contribution can be accounted for by two insertions of $e^{-2 \phi} G_{2}$. In this case, we just need to connect the vertex (4.26) for $M_{3}\left(1^{-}, 2^{-}, 3^{\varphi}\right)$ with its relabeled complex-conjugate,

$$
M_{3}^{\phi R^{2}}\left(1^{+}, 2^{+}, 3^{\varphi}\right)=-i[12]^{4},
$$

\footnotetext{
${ }^{5}$ The result in ref. [68] is written in a convention, where momenta are assumed to be ingoing for ingoing particles and outgoing for outgoing particles.
} 
via a scalar propagator in the $s$ channel. Again there are two contributing diagrams (from $\varphi$ and $\bar{\varphi}$ exchange), but this time only one of the three channels contributes. Thus one finds for the four-point gravity amplitude,

$$
M_{4}^{\left(\phi R^{2}\right)^{2}}\left(1^{-}, 2^{-}, 3^{+}, 4^{+}\right)=-2 i \frac{\langle 12\rangle^{4}[34]^{4}}{s_{12}} .
$$

Again the result all comes from dilaton exchange; the axion-exchange diagrams cancel. We used the KLT relations [1] to check that the $\mathcal{O}\left(\alpha^{\prime 2}\right)$ correction to the closed bosonic string tree-level amplitude indeed agrees with eq. (4.37).

Equation (4.37) is different from the previous examples in the following respect: In all previous cases, the amplitudes ' $M_{m}^{R+}$ ' generated by squaring $F^{3}$ amplitudes could be generated consistently from the $\mathcal{O}\left(\alpha^{2}\right)$ terms in the closed bosonic string effective action. But in this case it is not true, because $M_{4}^{R+}\left(1^{-}, 2^{-}, 3^{+}, 4^{+}\right)$vanishes according to eq. (4.35), while $M_{4}^{\left(\phi R^{2}\right)^{2}}$ does not. This discrepancy can be accounted for when we realize that at $\mathcal{O}\left(\alpha^{\prime 2}\right)$ in the string-theory KLT relations there are also contributions from Taylor expanding the sine functions to third order, and utilizing the leading YM terms in both open-string amplitudes. These contributions account for eq. (4.37) in the context of the KLT relations.

All of the above discussion was based on squaring amplitudes with one insertion of a vertex originating in $F^{3}$, for both the left- and right-moving gauge theory. However, the double-copy construction, as well as the KLT relations, in principle allow one to combine amplitudes originating in different theories and with different symmetries, provided that a color-kinematic dual representation is available for at least one of the two copies. For example, one- and two-loop amplitudes in $\mathcal{N}>4$ supergravities have been constructed in this way [14], and even more remarkably, the (vanishing) ultraviolet divergence in $\mathcal{N}=4$ supergravity at three loops [69].

Because dual representations are available for all gluon tree amplitudes in pure YangMills theory (and we have found the same to be true for all gluon amplitudes with one insertion of an $F^{3}$ vertex), we can combine YM with $F^{3}$ amplitudes in order to obtain pure-graviton amplitudes which would be at $\mathcal{O}\left(\alpha^{\prime}\right)$. However, we expect to get a vanishing result, because of an absence of potential operators, after field redefinitions and taking into account that the Gauss-Bonnet term is a total derivative in four dimensions.

For three and four external gravitons, the vanishing is trivial, because for those multiplicities there are no common nonzero helicity structures between pure YM and $F^{3}$ amplitudes, as has been shown in section 3. However, starting from the five-point level, helicity configurations with nonzero amplitudes of both kind exist. For example, the usual pure gauge-theory MHV helicity configuration $(--+++)$ coincides with the $\overline{\mathrm{MHV}}$ configuration from the $F^{3}$ sector. Thus, one can straightforwardly square those amplitudes into pure graviton ones. We have checked that the graviton amplitudes resulting from this mixed-KLT construction vanish for all helicity configurations at five and six points:

$$
\operatorname{KLT}_{\mathrm{FT}, m}\left[A_{m}, \tilde{A}_{m}^{F+}\right]=0, \quad m=5,6 .
$$

This vanishing result is just as expected from the form of the $\mathcal{O}\left(\alpha^{\prime}\right)$ term in eq. (4.19),

$$
e^{-2 \phi} G_{2}=\left[1-2 \phi+\mathcal{O}\left(\phi^{2}\right)\right] G_{2}
$$


because $G_{2}$ is a total derivative in four dimensions. Because it is purely topological, it cannot contribute to an amplitude. However, if one allows for external dilatons, this is not the case: a term like $-2 \phi G_{2}$ cannot be written as a total derivative and thus will contribute on shell, as we see already in the three-point amplitudes (4.26) and (4.36).

Thus the two sets of amplitudes, YM and $F^{3}$, are an example of a pairing, in which both sets satisfy color-kinematics duality, but the 'off-diagonal' double-copy construction, or KLT construction (4.38), does not yield nontrivial pure-graviton amplitudes. On the other hand, it does generate mixed scalar-graviton amplitudes, such as the three-point amplitude $M_{3}\left(1^{-}, 2^{-}, 3^{\varphi}\right)$ in eq. (4.26).

In summary, from the point of view of computing general $R^{3}$ amplitudes in gravity, the double-copy construction based on two sets of $F^{3}$ amplitudes does provide one ingredient. However, it would have to be combined with a separate computation of the effects of double insertions of $\phi R^{2}$ with dilaton exchange, as well as the effects of the YM-squared terms in the string-theory KLT relations, after expanding the sine functions to third order.

\section{Beyond $F^{3}$}

In this section, we briefly discuss the prospects for finding color-kinematic dual representations or BCJ amplitude relations for operators at dimension eight, built from four powers of the gluon field strength tensor $F_{\mu \nu}$. Once one combines more than three powers of the field strength, the index contraction pattern is no longer unique. At the next order, $F^{4}$ for short, there are four different contractions available (see e.g. refs. [34, 70]):

$$
\begin{array}{ll}
\operatorname{Tr}\left(F_{\mu}{ }^{\nu} F_{\nu}{ }^{\rho} F_{\rho}{ }^{\sigma} F_{\sigma}{ }^{\mu}\right), & \operatorname{Tr}\left(F_{\mu}{ }^{\nu} F_{\rho}{ }^{\sigma} F_{\nu}{ }^{\rho} F_{\sigma}{ }^{\mu}\right) \\
\operatorname{Tr}\left(F_{\mu \nu} F_{\rho \sigma} F^{\mu \nu} F^{\rho \sigma}\right), & \operatorname{Tr}\left(F_{\mu \nu} F^{\mu \nu} F_{\rho \sigma} F^{\rho \sigma}\right) .
\end{array}
$$

(As at dimension six in pure YM, terms with covariant derivatives are removable by using field redefinitions.) In pure gauge theory, any of these insertions can occur separately with an independent coefficient. However, if the theory is supersymmetric, the possible coefficients are constrained. The particular unique linear combination appearing at $\mathcal{O}\left(\alpha^{\prime 2}\right)$ in the low-energy effective action of the open superstring (ss), corresponding to $\mathcal{N}=4$ supersymmetry, is [70-72]:

$$
\begin{aligned}
F_{\mathrm{ss}}^{4} \equiv \operatorname{Tr}[ & F_{\mu}{ }^{\nu} F_{\nu}{ }^{\rho} F_{\rho}{ }^{\sigma} F_{\sigma}{ }^{\mu}+2 F_{\mu}{ }^{\nu} F_{\rho}{ }^{\sigma} F_{\nu}{ }^{\rho} F_{\sigma}{ }^{\mu} \\
& \left.\quad-\frac{1}{4} F_{\mu \nu} F_{\rho \sigma} F^{\mu \nu} F^{\rho \sigma}-\frac{1}{2} F_{\mu \nu} F^{\mu \nu} F_{\rho \sigma} F^{\rho \sigma}\right] .
\end{aligned}
$$

Amplitudes originating from insertions of operators appearing in the string effective action can be derived from the infinite-tension limit $\alpha^{\prime} \rightarrow 0$ of the corresponding string amplitude. After factoring out the field-theory MHV amplitude, the expansion for the four-gluon amplitude reads $[73]:^{6}$

$$
A_{\mathrm{ss}}\left(1^{-}, 2^{-}, 3^{+}, 4^{+}\right)=i \frac{\langle 12\rangle^{4}}{\langle 12\rangle\langle 23\rangle\langle 34\rangle\langle 41\rangle}\left[1-\alpha^{\prime 2} \zeta(2) s t+\alpha^{\prime 3} \zeta(3) s t u+\mathcal{O}\left(\alpha^{\prime 4}\right)\right],
$$

\footnotetext{
${ }^{6}$ In ref. [73] another metric signature is used, which accounts for the sign difference with respect to the expression there.
} 
while expressions for higher multiplicity are available from the same reference. A short calculation reveals that amplitudes at $\mathcal{O}\left(\alpha^{\prime 2}\right)$ in the superstring expansion do not allow for a color-kinematic dual representation. In order to find the reason for this behavior, let us consider the string-theory monodromy relations once again [3-5]. For simplicity we will write the relations at four points, however, higher multiplicities work analogously. The four-point form of eq. (3.34) relating color-ordered subamplitudes of the string tree-level amplitude reads:

$$
A^{\text {string }}(1,2,3,4)+e^{i \pi \alpha^{\prime} s} A^{\text {string }}(2,1,3,4)+e^{i \pi \alpha^{\prime}(s+u)} A^{\text {string }}(2,3,1,4)=0 .
$$

In order to obtain relations between different parts of the string amplitude appearing at different orders in $\alpha^{\prime}$, one needs to expand the string amplitude $A^{\text {string }}$ via

$$
A^{\text {string }}=A^{\mathrm{YM}}+\alpha^{\prime} A^{\alpha^{\prime}}+\alpha^{\prime 2} A^{\alpha^{\prime 2}}+\alpha^{\prime 3} A^{\alpha^{\prime 3}}+\cdots .
$$

Also expanding the exponential, one finds that the monodromy relations at the first few orders in $\alpha^{\prime}$ read: ${ }^{7}$

$$
\begin{aligned}
\mathcal{O}\left(\alpha^{\prime 0}\right): \quad 0= & A^{\mathrm{YM}}(1,2,3,4)+A^{\mathrm{YM}}(2,1,3,4)+A^{\mathrm{YM}}(2,3,1,4), \\
\mathcal{O}\left(\alpha^{\prime 1}\right): \quad 0= & s A^{\mathrm{YM}}(2,1,3,4)-t A^{\mathrm{YM}}(2,3,1,4), \\
\mathcal{O}\left(\alpha^{\prime 1}\right): \quad 0= & A^{\alpha^{\prime}}(1,2,3,4)+A^{\alpha^{\prime}}(2,1,3,4)+A^{\alpha^{\prime}}(2,3,1,4), \\
\mathcal{O}\left(\alpha^{\prime 2}\right): \quad 0= & s A^{\alpha^{\prime}}(2,3,1,4)-t A^{\alpha^{\prime}}(2,1,3,4), \\
\mathcal{O}\left(\alpha^{\prime 2}\right): \quad 0=- & \frac{\pi^{2}}{2} s^{2} A^{\mathrm{YM}}(2,3,1,4)-\frac{\pi^{2}}{2} t^{2} A^{\mathrm{YM}}(2,1,3,4) \\
& +A^{\alpha^{\prime 2}}(1,2,3,4)+A^{\alpha^{\prime 2}}(2,1,3,4)+A^{\alpha^{\prime 2}}(2,3,1,4), \\
\mathcal{O}\left(\alpha^{\prime 3}\right): \quad 0=- & \frac{\pi^{2}}{6} s^{3} A^{\mathrm{YM}}(2,1,3,4)+\frac{\pi^{2}}{6} t^{3} A^{\mathrm{YM}}(2,3,1,4) \\
& +s A^{\alpha^{\prime 2}}(2,1,3,4)-t A^{\alpha^{\prime 2}}(2,3,1,4), \\
\mathcal{O}\left(\alpha^{\prime 3}\right): \quad 0=- & \frac{\pi^{2}}{2} s^{2} A^{\alpha^{\prime}}(2,1,3,4)-\frac{\pi^{2}}{2} t^{2} A^{\alpha^{\prime}}(2,3,1,4) \\
& +A^{\alpha^{\prime 3}}(1,2,3,4)+A^{\alpha^{\prime 3}}(2,1,3,4)+A^{\alpha^{\prime 3}}(2,3,1,4) .
\end{aligned}
$$

These relations are valid for supersymmetric as well as bosonic string theory. In the former case, the expressions can be simplified by noticing that $A^{\alpha^{\prime}}$ vanishes.

The first two lines in the above expansions, eqs. (5.6) and (5.7), yield the photon decoupling and BCJ relations for the YM amplitudes, while eqs. (5.8) and (5.9) manifest the same relations for the amplitudes $A^{\alpha^{\prime}}$, which are equivalent to the $F^{3}$ amplitudes. Equation (5.9) is one example of the BCJ amplitude representations obtained for all $F^{3}$ amplitudes in section 3 .

The second part of eq. (5.10) has the same form as the photon-decoupling equation in the lines above, but for $A^{\alpha^{\prime 2}}$. However, this relation is spoiled by the two other terms, given by Mandelstam variables multiplying the YM amplitudes. Thus, one should not

\footnotetext{
${ }^{7}$ For completeness we have also included the relations already appearing in eqs. (3.38) and (3.39).
} 
expect the amplitudes at $\mathcal{O}\left(\alpha^{\prime 2}\right)$ in the bosonic string or the superstring to satisfy the usual photon-decoupling identity or BCJ relations (i.e. eq. (5.11) also has extra terms).

Indeed, considering the collection of eqs. (2.5) and (2.12), together with the amplitude equations, e.g.,

$$
A^{\alpha^{\prime 2}}(1,2,3,4)=\frac{n_{s}}{s}+\frac{n_{t}}{t},
$$

and the corresponding ones for $A^{\alpha^{\prime 2}}(1,2,4,3)$ and $A^{\alpha^{\prime 2}}(3,1,4,2)$, the system does not have nontrivial solutions for any helicity structure, neither in bosonic nor in superstring theory.

Is there a way to explain this behavior in terms of the color structure accompanying the corresponding higher-derivative operators at the appropriate orders in $\alpha^{\prime}$ ? For the simple example of the four-point YM tree amplitude $\left(\mathcal{O}\left(\alpha^{\prime 0}\right)\right)$ in section 2, the symmetries of the kinematic part allowed rewriting the trace-based amplitude decomposition in terms of the totally antisymmetric structure constants $f^{a b c}$ only. (Of course this must be possible, since the YM Feynman rules are expressed in this way.)

For the insertion of the unique dimension-six operator $F^{3}$ this property was already shown at the level of the action (see eq. (3.2)): One can decompose a trace of three matrices into symmetric and antisymmetric parts,

$$
\operatorname{Tr}\left(T^{a} T^{b} T^{c}\right)=d^{a b c}+\frac{1}{2} \tilde{f}^{a b c} .
$$

Here $d^{a b c}$ is the case $n=3$ of the more general totally symmetric trace structure, summed over all $n$ ! permutations $\mathcal{P}\left(a_{1}, \ldots, a_{n}\right)$,

$$
d^{a_{1} \ldots a_{n}}=\frac{1}{n !} \sum_{\sigma \in \mathcal{P}\left(a_{1}, \ldots, a_{n}\right)} \operatorname{Tr}\left(T^{a_{\sigma(1)}} \ldots T^{a_{\sigma(n)}}\right) .
$$

However, for $F^{3}$ the antisymmetry of the field strength $F_{\mu \nu}$ removes the symmetric part, leaving only the totally antisymmetric part $\tilde{f}^{a b c}$.

In contrast, for the open superstring at $\mathcal{O}\left(\alpha^{2}\right)$ the operator $F_{\mathrm{sS}}^{4}$ in eq. (5.2) projects onto the totally symmetric part of the space of four-color-matrix traces [74, 75]:

$$
\operatorname{Tr}\left(T^{a} T^{b} T^{c} T^{d}\right)\left(F_{\mathrm{ss}}^{4}\right)_{a b c d} \propto d^{a b c d}\left(F_{\mathrm{ss}}^{4}\right)_{a b c d},
$$

where the general color-trace can be decomposed as,

$$
\operatorname{Tr}\left(T^{a} T^{b} T^{c} T^{d}\right)=d^{a b c d}+\frac{1}{2}\left(\tilde{f}^{b c e} d^{e a d}-\tilde{f}^{a d e} d^{e b c}\right)-\frac{1}{6}\left(\tilde{f}^{a d e} \tilde{f}^{e b c}-\tilde{f}^{a b e} \tilde{f}^{e c d}\right) .
$$

It is not possible to write $d^{a b c d}$ purely in terms of antisymmetric structure constants $\tilde{f}^{a b c}$. Because the Kleiss-Kuijf and, in particular, the photon-decoupling identity are proven by writing amplitudes in terms of structure constants, it is no surprise that the amplitudes $A^{\alpha^{\prime 2}}$ generated by $F_{\mathrm{ss}}^{4}$ do not even satisfy photon-decoupling, eq. (5.10). Therefore this combination of $F^{4}$ operators is not color-compatible with a color-kinematics duality based on cubic graphs.

In order to search for combinations of $F^{4}$ operators that are color-compatible, we first recognize that eqs. (5.10) and (5.11) are valid for the open bosonic string (bs) and the 
open superstring (ss) at the same time, and that the YM amplitudes are the same in both theories. Therefore if we take the difference (diff) of the two sets of amplitudes,

$$
A_{\mathrm{diff}}^{\alpha^{\prime 2}}=A_{\mathrm{bs}}^{\alpha^{\prime 2}}-A_{\mathrm{ss}}^{\alpha^{\prime 2}}
$$

then the differences of the corresponding eqs. (5.10) and (5.11) will remove the common YM terms that were spoiling the photon-decoupling and BCJ relations at $\mathcal{O}\left(\alpha^{2}\right)$ :

$$
\begin{aligned}
& 0=A_{\mathrm{diff}}^{\alpha^{\prime 2}}(1,2,3,4)+A_{\mathrm{diff}}^{\alpha^{\prime 2}}(2,1,3,4)+A_{\mathrm{diff}}^{\alpha^{\prime 2}}(2,3,1,4) \\
& 0=s A_{\mathrm{diff}}^{\alpha^{\prime 2}}(2,1,3,4)-t A_{\mathrm{diff}}^{\alpha^{\prime 2}}(2,3,1,4) .
\end{aligned}
$$

Thus, one should be able to express the difference between the bosonic and superstring effective actions at $\mathcal{O}\left(\alpha^{\prime 2}\right)$ in terms of operators that are color-compatible (built only out of $\left.\tilde{f}^{a b c} \mathrm{~s}\right)$.

The two obvious $F^{4}$-type operators with color structure $\tilde{f}^{a b e} \tilde{f}^{c d e}$ are

$$
O_{A}=\operatorname{Tr}\left(\left[F_{\mu}{ }^{\nu}, F_{\nu}{ }^{\rho}\right]\left[F_{\rho}{ }^{\sigma}, F_{\sigma}{ }^{\mu}\right]\right) \quad \text { and } \quad O_{B}=\operatorname{Tr}\left(\left[F_{\mu \nu}, F_{\rho \sigma}\right]\left[F^{\mu \nu}, F^{\rho \sigma}\right]\right)
$$

These operators are linear combinations of the operators given in eq. (5.1). Their contributions to four-point amplitudes can be determined easily. Amplitudes generated by both operators necessarily satisfy the photon-decoupling identity. On the other hand, neither operator, nor any linear combination of $O_{A}$ and $O_{B}$, generates amplitudes that satisfy the BCJ amplitude relations. Thus a color-kinematic dual representation is impossible. How can this be, given that eq. (5.20) looks like a BCJ relation?

The explanation is similar to that for the closed-string discrepancy between $M_{4}^{R+}\left(1^{-}, 2^{-}, 3^{-}, 4^{-}\right)$and $M_{4}^{R^{3}}\left(1^{-}, 2^{-}, 3^{-}, 4^{-}\right)$discussed in section 4: One has to take into account two insertions of a lower-dimension operator. In this case, it is the operator $F^{3}$ at order $\alpha^{\prime}$, and gluons are exchanged between the two insertions. Performing the appropriate Feynman-diagram calculation leads to a third contribution at $\mathcal{O}\left(\alpha^{\prime 2}\right)$, denoted by $A^{\left(F^{3}\right)^{2}}$. Not surprisingly, the contribution $A^{\left(F^{3}\right)^{2}}$ alone satisfies photon-decoupling, but not the BCJ relations. However, a specific linear combination of $A^{O_{A}}, A^{O_{B}}$ and $A^{\left(F^{3}\right)^{2}}$ correctly reproduces the amplitude difference $A_{\text {diff }}^{\alpha^{\prime 2}}$ between the bosonic string and the superstring:

$$
A_{\mathrm{bs}}^{\alpha^{\prime 2}}-A_{\mathrm{ss}}^{\alpha^{\prime 2}}=A_{\mathrm{diff}}^{\alpha^{\prime 2}}=\frac{1}{2} A^{\left(F^{3}\right)^{2}}+\frac{1}{4} A^{O_{B}}
$$

Expanding the four-point amplitude for the bosonic string (Veneziano amplitude) to $\mathcal{O}\left(\alpha^{2}\right)$ and subtracting the superstring result (5.3) gives,

$$
\begin{aligned}
& A_{\mathrm{diff}}^{\alpha^{\prime 2}}\left(1^{-}, 2^{-}, 3^{+}, 4^{+}\right)=-u t A^{\mathrm{YM}}\left(1^{-}, 2^{-}, 3^{+}, 4^{+}\right), \\
& A_{\mathrm{diff}}^{\alpha^{\prime 2}}\left(1^{-}, 2^{+}, 3^{-}, 4^{+}\right)=- \text {st } A^{\mathrm{YM}}\left(1^{-}, 2^{+}, 3^{-}, 4^{+}\right) .
\end{aligned}
$$


The corresponding contributions from $\left(F^{3}\right)^{2}$ and $O_{B}$ are given by,

$$
\begin{aligned}
\frac{1}{2} A^{\left(F^{3}\right)^{2}}\left(1^{-}, 2^{-}, 3^{+}, 4^{+}\right) & =\frac{t^{2}-u t}{2} A^{\mathrm{YM}}\left(1^{-}, 2^{-}, 3^{+}, 4^{+}\right), \\
\frac{1}{4} A^{O_{B}}\left(1^{-}, 2^{-}, 3^{+}, 4^{+}\right) & =\frac{s t}{2} A^{\mathrm{YM}}\left(1^{-}, 2^{-}, 3^{+}, 4^{+}\right), \\
\frac{1}{2} A^{\left(F^{3}\right)^{2}}\left(1^{-}, 2^{+}, 3^{-}, 4^{+}\right) & =0, \\
\frac{1}{4} A^{O_{B}}\left(1^{-}, 2^{+}, 3^{-}, 4^{+}\right) & =-s t A^{\mathrm{YM}}\left(1^{-}, 2^{+}, 3^{-}, 4^{+}\right) .
\end{aligned}
$$

Using the fact that $s t A^{\mathrm{YM}}$ is totally symmetric, and factoring this quantity out, it is easy to see that $A_{\mathrm{diff}}^{\alpha^{\prime 2}}$ satisfies photon decoupling,

$$
\begin{aligned}
& A_{\mathrm{diff}}^{\alpha^{\prime 2}}\left(1^{-}, 2^{-}, 3^{+}, 4^{+}\right)+A_{\mathrm{diff}}^{\alpha^{\prime 2}}\left(2^{-}, 1^{-}, 3^{+}, 4^{+}\right)+A_{\mathrm{diff}}^{\alpha^{\prime 2}}\left(2^{-}, 3^{+}, 1^{-}, 4^{+}\right) \\
& \quad=s t A^{\mathrm{YM}}\left(1^{-}, 2^{-}, 3^{+}, 4^{+}\right)\left[\frac{u}{s}+\frac{t}{s}+1\right]=0
\end{aligned}
$$

and the BCJ relation,

$$
s A_{\mathrm{diff}}^{\alpha^{\prime 2}}\left(2^{-}, 1^{-}, 3^{+}, 4^{+}\right)-t A_{\mathrm{diff}}^{\alpha^{\prime 2}}\left(2^{-}, 3^{+}, 1^{-}, 4^{+}\right)=s t A^{\mathrm{YM}}\left(1^{-}, 2^{-}, 3^{+}, 4^{+}\right)\left[s \frac{t}{s}-t\right]=0 .
$$

Similarly, $A^{O_{B}}$ obeys photon decoupling,

$$
\begin{aligned}
& A^{O_{B}}\left(1^{-}, 2^{-}, 3^{+}, 4^{+}\right)+A^{O_{B}}\left(2^{-}, 1^{-}, 3^{+}, 4^{+}\right)+A^{O_{B}}\left(2^{-}, 3^{+}, 1^{-}, 4^{+}\right) \\
& \quad=s t A^{\mathrm{YM}}\left(1^{-}, 2^{-}, 3^{+}, 4^{+}\right)[2+2-4]=0,
\end{aligned}
$$

but the BCJ relation does not hold,

$$
s A^{O_{B}}\left(2^{-}, 1^{-}, 3^{+}, 4^{+}\right)-t A^{O_{B}}\left(2^{-}, 3^{+}, 1^{-}, 4^{+}\right)=s t A^{\mathrm{YM}}\left(1^{-}, 2^{-}, 3^{+}, 4^{+}\right)[2 s-4 t] \neq 0 .
$$

The above example shows that the correct color structure alone is not a sufficient condition for the existence of a color-kinematic dual representation. In addition, one needs the right kinematic behavior, which in this case could only happen if one combines two insertions of the dimension-six operator $F^{3}$ and one insertion of the dimension-eight operator $O_{B}$ with the right relative coefficient.

We also tested higher-order in $\alpha^{\prime}$ contributions to the superstring amplitude. Except for $\mathcal{O}\left(\alpha^{\prime 3}\right)$, we have not found color-kinematic dual representations to exist for even the fourpoint amplitude. At $\mathcal{O}\left(\alpha^{\prime 3}\right)$, the four-gluon amplitude does obey the photon-decoupling and BCJ relations. Therefore we have examined the five- and six-gluon amplitudes as well, using the open-string amplitudes from refs. [73, 76]; we find that they obey both sets of equations. The effective action at this order is a particular linear combination of operators of the type $D^{2} F^{4}$ and $F^{5}$, where $D$ denotes a gauge-covariant derivative [74].

These results nicely match the known color structure of the four-point amplitude [74]. The four-point superstring amplitude can be represented as

$$
\left.\mathcal{A}(1,2,3,4)\right|_{\alpha^{\prime} \rightarrow 0}=K(1,2,3,4) \sum_{n=0}^{\infty} \alpha^{\prime n} a^{(n)},
$$




\begin{tabular}{|c|c|}
\hline$a^{(0)}$ & $-\frac{1}{2 s}\left(\frac{1}{u} \tilde{f}^{a c e} \tilde{f}^{b d e}+\frac{1}{t} \tilde{f}^{a d e} \tilde{f}^{b c e}\right)$ \\
\hline$a^{(1)}$ & 0 \\
\hline$a^{(2)}$ & $-6 \zeta(2) d^{a b c d}$ \\
\hline$a^{(3)}$ & $-\frac{\zeta(3)}{2}\left(u \tilde{f}^{a b e} \tilde{f}^{c d e}+s \tilde{f}^{a c e} \tilde{f} b d e\right)$ \\
\hline$a^{(4)}$ & $-\frac{3}{2} \zeta(2)^{2}\left(s^{2}+t^{2}+u^{2}\right) d^{a b c d}$ \\
& $-\frac{\zeta(4)}{8}\left[s(u-t) \tilde{f}^{a b e} \tilde{f}^{c d e}+u(s-t) \tilde{f}^{a c e} \tilde{f}^{b d e}+t(s-u) \tilde{f}^{a d e} \tilde{f}^{b c e}\right]$ \\
\hline
\end{tabular}

Table 1. Corrections to the tree-level four-point gauge-theory amplitude from the low-energy expansion of open superstring theory [74].

where the prefactor $K(1,2,3,4)$ describes the complete state dependence and $a^{(n)}$ is a totally symmetric contribution containing the color dependence at $\mathcal{O}\left(\alpha^{\prime n}\right)$. Considering the factors in table 1 from ref. [74] (which contains results through $\mathcal{O}\left(\alpha^{\prime 6}\right)$ ), one can see that (with the exception of $\mathcal{O}\left(\alpha^{\prime 3}\right)$ ) they are not purely built from $\tilde{f}^{a b c} \mathrm{~s}$, but contain totally symmetric $d^{a b c d}$ contributions. Thus it is not surprising that one cannot find tree-level dual representations for stringy $\alpha^{\prime}$ corrections beyond $\mathcal{O}\left(\alpha^{\prime 3}\right)$. Finally, we note that the $\mathcal{O}\left(\alpha^{\prime 3}\right)$ case, for which we do have evidence of color-kinematics duality through at least six external states, does not suffer from multiple insertions of lower-dimension operators, because there is no $\mathcal{O}\left(\alpha^{\prime}\right)$ correction to the superstring amplitude. The first case of multiple insertions is delayed until $\mathcal{O}\left(\alpha^{\prime 4}\right)$.

Another area in which one might search for color-kinematics duality is in 'form factors', that is, the matrix elements of operators $O(x)$ with plane-wave states in gauge theory. In momentum space, generic momentum is injected at the operator. Perhaps the simplest case to consider is $O=\operatorname{Tr}\left(F^{\mu \nu} F_{\mu \nu}\right)$ or $\tilde{O}=\operatorname{Tr}\left(F^{\mu \nu} \tilde{F}_{\mu \nu}\right)$. The injected momentum can be thought of as carried by a scalar or pseudoscalar particle. For $O$ (or $\tilde{O}$ ), the coupling to gluons is the same as for the Standard Model Higgs boson (or its pseudoscalar partner $A$ in a two-Higgs doublet model) in the heavy top-quark limit, through the coupling $H \operatorname{Tr}\left(F^{\mu \nu} F_{\mu \nu}\right)$ (or $A \operatorname{Tr}\left(F^{\mu \nu} \tilde{F}_{\mu \nu}\right)$ ). Matrix elements for a Higgs boson coupling to multiple gluons have been studied often, for example in ref. [29].

Here we simply wish to ask whether these matrix elements might exhibit colorkinematics duality. At first sight, one might be optimistic, because the class of MHV amplitudes,

$$
A_{m}\left(\phi, 1^{+}, 2^{+}, \ldots, p^{-}, \ldots, q^{-}, \ldots, m^{+}\right)
$$

where $\phi=\frac{1}{2}(H+i A)$, has precisely the same formula [29] as the pure YM Parke-Taylor amplitudes (3.8). However, when one checks the BCJ amplitude relation for four gluons, using this formula, one finds that it is spoiled by the momentum injected by the $\phi$ particle. The photon-decoupling relation continues to hold, however, for all values of $m>2$. The latter holds because the color-dressed $\phi$-amplitudes $\mathcal{A}_{m}(\phi, \ldots)$ for $m>2$ can be expressed solely in terms of structure constants $\tilde{f}^{a b c}$. 
However, the $m=2$ amplitude is exceptional: It is proportional to $\delta^{a_{1} a_{2}}$,

$$
\mathcal{A}_{2}\left(\phi, 1^{-}, 2^{-}\right) \propto \delta^{a_{1} a_{2}}\langle 12\rangle^{2} .
$$

Hence the color-ordered amplitude $A_{2}\left(\phi, 1^{-}, 2^{-}\right)$does not even obey photon-decoupling. Heuristically, we can attribute the failure of the BCJ amplitude relations for $m>2$ to the mismatch between color and kinematics in a cubic-graph representation: There should be a $\phi g g$ kinematical vertex to account for the momentum injected by $\phi$, but there is no corresponding $\tilde{f}^{a b c}$ color factor.

\section{Conclusions and outlook}

In this article we studied whether color-kinematics duality in gauge theory can be extended beyond the renormalizable level, deforming the pure YM theory by higher-dimension operators composed from the gluon field strength. Using string-theory monodromy relations, we found that the amplitudes produced by one insertion of the operator $F^{3}$ obey BCJ amplitude relations. We verified these relations for a variety of multiplicities and helicity configurations, using CSW rules to construct the amplitudes (in perfect numerical agreement with a Feynman-diagram calculation using MADGRAPH). We also determined (often numerically) the existence of color-kinematic dual representations for all these cases.

The double copy of those representations yields amplitudes which originate in corrections to Einstein gravity present in the effective action of the closed bosonic string up to order $\mathcal{O}\left(\alpha^{\prime 2}\right)$. Besides diagrams involving an $R^{3}$ operator and gravitons on internal lines only, one also has to consider dilaton-exchange diagrams involving two insertions of a $\phi R^{2}$ operator. The effects of YM-squared terms in the string-theory KLT relations, after expanding the sine functions of the momentum invariants to third order, have to be considered as well in some cases.

Our results show - up to the order tested - that the double-copy construction can be equivalent to the KLT relations at higher orders in the inverse string tension $\alpha^{\prime}$. In addition to squaring the modified amplitudes and checking the gravity amplitudes for Bose symmetry, collinearity and soft limits, we implemented all-line BCFW shifts for suitable $R^{3}$ gravity amplitudes as an additional test.

For several other additions of operators to the Yang-Mills action, tree-level colorkinematic dual representations do not exist. In some cases we could attribute this property to the color structure of the operator. If the operator is not composed entirely from totally antisymmetric structure constants $f^{a b c}$ (as in examples drawn from the low-energy effective action of open string theory), then a standard dual representation will not exist. In fact, the photon-decoupling identities will not be obeyed. Whether a generalized color-kinematics duality could hold in some of these cases, involving four- or higher-index structure constants [17], is a question that is certainly deserving of future investigation. Another avenue for further work could be to investigate higher-dimension operators in gauge theory that include matter fields as well as gauge fields.

We also found that even some operators that are color-compatible with the usual colorkinematics duality do not actually lead to amplitudes obeying it, such as the dimension- 
eight $F^{4}$-type operators $O_{A}$ and $O_{B}$ studied in section 5 . Thus, color structure alone is not sufficient to ensure color-kinematics duality. However, one of these operators, $O_{B}$, can be combined with two insertions of $F^{3}$ in order to restore color-kinematics duality. This combination corresponds to the difference of terms in the bosonic and superstring effective actions. Also, the $\mathcal{O}\left(\alpha^{\prime 3}\right)$ term in the superstring effective action appears to generate amplitudes consistent with color-kinematics duality, at least through six external gluons. The above results hint that color-kinematics duality may be closely related to string theory also beyond the leading order in $\alpha^{\prime}$; however, more detailed work would be needed to fully explore these connections. The recent motivic organization of superstring amplitudes [77] may provide very useful clues in this regard.

\section{Acknowledgments}

We thank Zvi Bern, Rutger Boels, John Joseph Carrasco, Tim Cohen, Henriette Elvang, Michael Kiermaier and Radu Roiban for stimulating discussions and correspondence. J.B. is indebted to Claude Duhr for a lightning introduction to the use of MADGRAPH and for providing the MADGRAPH implementation for $F^{3}$ vertices via FEYNRULES. This work was supported in part by the US Department of Energy under contract DE-AC02-76SF00515. J.B. gratefully acknowledges support from the Alexander-von-Humboldt foundation within the Feodor-Lynen program.

Open Access. This article is distributed under the terms of the Creative Commons Attribution License which permits any use, distribution and reproduction in any medium, provided the original author(s) and source are credited.

\section{References}

[1] H. Kawai, D. Lewellen and S. Tye, A relation between tree amplitudes of closed and open strings, Nucl. Phys. B 269 (1986) 1 [INSPIRE].

[2] Z. Bern, J. Carrasco and H. Johansson, New relations for gauge-theory amplitudes, Phys. Rev. D 78 (2008) 085011 [arXiv:0805.3993] [InSPIRE].

[3] N. Bjerrum-Bohr, P.H. Damgaard and P. Vanhove, Minimal basis for gauge theory amplitudes, Phys. Rev. Lett. 103 (2009) 161602 [arXiv:0907.1425] [INSPIRE].

[4] S. Stieberger, Open \& closed vs. pure open string disk amplitudes, arXiv:0907.2211 [INSPIRE].

[5] N. Bjerrum-Bohr, P.H. Damgaard, T. Sondergaard and P. Vanhove, Monodromy and Jacobi-like relations for color-ordered amplitudes, JHEP 06 (2010) 003 [arXiv:1003.2403] [INSPIRE].

[6] B. Feng, R. Huang and Y. Jia, Gauge amplitude identities by on-shell recursion relation in S-matrix program, Phys. Lett. B 695 (2011) 350 [arXiv:1004.3417] [INSPIRE].

[7] R. Britto, F. Cachazo and B. Feng, New recursion relations for tree amplitudes of gluons, Nucl. Phys. B 715 (2005) 499 [hep-th/0412308] [INSPIRE].

[8] R. Britto, F. Cachazo, B. Feng and E. Witten, Direct proof of tree-level recursion relation in Yang-Mills theory, Phys. Rev. Lett. 94 (2005) 181602 [hep-th/0501052] [INSPIRE]. 
[9] Z. Bern, T. Dennen, Y.-t. Huang and M. Kiermaier, Gravity as the square of gauge theory, Phys. Rev. D 82 (2010) 065003 [arXiv: 1004.0693] [inSPIRE].

[10] Z. Bern, J.J.M. Carrasco and H. Johansson, Perturbative quantum gravity as a double copy of gauge theory, Phys. Rev. Lett. 105 (2010) 061602 [arXiv:1004.0476] [INSPIRE].

[11] J.J. Carrasco and H. Johansson, Five-point amplitudes in $N=4$ super-Yang-Mills theory and $N=8$ supergravity, Phys. Rev. D 85 (2012) 025006 [arXiv:1106.4711] [INSPIRE].

[12] J.J.M. Carrasco and H. Johansson, Generic multiloop methods and application to $N=4$ super-Yang-Mills, J. Phys. A 44 (2011) 454004 [arXiv:1103.3298] [InSPIRE].

[13] Z. Bern, C. Boucher-Veronneau and H. Johansson, $N \geq 4$ supergravity amplitudes from gauge theory at one loop, Phys. Rev. D 84 (2011) 105035 [arXiv:1107.1935] [INSPIRE].

[14] C. Boucher-Veronneau and L. Dixon, $N \geq 4$ supergravity amplitudes from gauge theory at two loops, JHEP 12 (2011) 046 [arXiv:1110.1132] [INSPIRE].

[15] S.G. Naculich, H. Nastase and H.J. Schnitzer, Linear relations between $N \geq 4$ supergravity and subleading-color SYM amplitudes, JHEP 01 (2012) 041 [arXiv:1111.1675] [INSPIRE].

[16] Z. Bern, J. Carrasco, L. Dixon, H. Johansson and R. Roiban, Simplifying multiloop integrands and ultraviolet divergences of gauge theory and gravity amplitudes, Phys. Rev. D 85 (2012) 105014 [arXiv:1201.5366] [inSPIRE].

[17] T. Bargheer, S. He and T. McLoughlin, New relations for three-dimensional supersymmetric scattering amplitudes, Phys. Rev. Lett. 108 (2012) 231601 [arXiv: 1203.0562] [INSPIRE].

[18] J. Bagger and N. Lambert, Modeling multiple M2's, Phys. Rev. D 75 (2007) 045020 [hep-th/0611108] [INSPIRE].

[19] A. Gustavsson, Algebraic structures on parallel M2-branes, Nucl. Phys. B 811 (2009) 66 [arXiv: 0709.1260] [INSPIRE].

[20] J. Bagger and N. Lambert, Gauge symmetry and supersymmetry of multiple M2-branes, Phys. Rev. D 77 (2008) 065008 [arXiv:0711.0955] [InSPIRE].

[21] J. Polchinski, String theory, Cambridge University Press, Cambridge U.K. (1998).

[22] P. Benincasa and E. Conde, Exploring the S-matrix of massless particles, Phys. Rev. D 86 (2012) 025007 [arXiv:1108.3078] [InSPIRE].

[23] E.H. Simmons, Dimension-six gluon operators as probes of new physics, Phys. Lett. B 226 (1989) 132 [INSPIRE].

[24] E.H. Simmons, Higher dimension gluon operators and hadronic scattering, Phys. Lett. B 246 (1990) 471 [INSPIRE].

[25] P.L. Cho and E.H. Simmons, Looking for gluon substructure at the Tevatron, Phys. Lett. B 323 (1994) 401 [hep-ph/9307345] [INSPIRE].

[26] A. Duff and D. Zeppenfeld, Probing QCD via four jet decays of the $Z$ boson, Z. Phys. C 53 (1992) 529 [INSPIRE].

[27] H.K. Dreiner, A. Duff and D. Zeppenfeld, How well do we know the three gluon vertex?, Phys. Lett. B 282 (1992) 441 [INSPIRE].

[28] L.J. Dixon and Y. Shadmi, Testing gluon selfinteractions in three jet events at hadron colliders, Nucl. Phys. B 423 (1994) 3 [Erratum ibid. B 452 (1995) 724] [hep-ph/9312363] [INSPIRE]. 
[29] L.J. Dixon, E.W.N. Glover and V.V. Khoze, MHV rules for Higgs plus multi-gluon amplitudes, JHEP 12 (2004) 015 [hep-th/0411092] [INSPIRE].

[30] T. Cohen, H. Elvang and M. Kiermaier, On-shell constructibility of tree amplitudes in general field theories, JHEP 04 (2011) 053 [arXiv: 1010.0257] [INSPIRE].

[31] D. Neill, Analytic Virtual Corrections for Higgs Transverse Momentum Spectrum at $O\left(\alpha_{s}^{2} / m_{t}^{3}\right)$ via Unitarity Methods, arXiv:0911.2707 [INSPIRE].

[32] F. Cachazo, P. Svrček and E. Witten, MHV vertices and tree amplitudes in gauge theory, JHEP 09 (2004) 006 [hep-th/0403047] [INSPIRE].

[33] R. Boels and C. Schwinn, Deriving CSW rules for massive scalar legs and pure Yang-Mills loops, JHEP 07 (2008) 007 [arXiv:0805.1197] [INSPIRE].

[34] N. Bjerrum-Bohr, String theory and the mapping of gravity into gauge theory, Phys. Lett. B 560 (2003) 98 [hep-th/0302131] [INSPIRE].

[35] N. Bjerrum Bohr, Generalized string theory mapping relations between gravity and gauge theory, Nucl. Phys. B 673 (2003) 41 [hep-th/0305062] [INSPIRE].

[36] Z. Bern and T. Dennen, A color dual form for gauge-theory amplitudes, Phys. Rev. Lett. 107 (2011) 081601 [arXiv:1103.0312] [inSPIRE].

[37] D.-p. Zhu, Zeros in scattering amplitudes and the structure of non-Abelian gauge theories, Phys. Rev. D 22 (1980) 2266 [INSPIRE].

[38] C. Goebel, F. Halzen and J. Leveille, Angular zeros of Brown, Mikaelian, Sahdev and Samuel and the factorization of tree amplitudes in gauge theories, Phys. Rev. D 23 (1981) 2682 [INSPIRE].

[39] R. Kleiss and H. Kuijf, Multi-gluon cross-sections and five jet production at hadron colliders, Nucl. Phys. B 312 (1989) 616 [inSPIRE].

[40] V. Del Duca, L.J. Dixon and F. Maltoni, New color decompositions for gauge amplitudes at tree and loop level, Nucl. Phys. B 571 (2000) 51 [hep-ph/9910563] [INSPIRE].

[41] M. Kiermaier, Gravity as the Square of Gauge Theory, presented at Amplitudes 2010, London U.K. (2010), http://www.strings.ph.qmul.ac.uk/ theory/Amplitudes2010.

[42] N. Bjerrum-Bohr, P.H. Damgaard, T. Sondergaard and P. Vanhove, The momentum kernel of gauge and gravity theories, JHEP 01 (2011) 001 [arXiv: 1010.3933] [INSPIRE].

[43] C.R. Mafra, O. Schlotterer and S. Stieberger, Explicit BCJ numerators from pure spinors, JHEP 07 (2011) 092 [arXiv: 1104.5224] [INSPIRE].

[44] R. Monteiro and D. O'Connell, The kinematic algebra from the self-dual sector, JHEP 07 (2011) 007 [arXiv:1105.2565] [INSPIRE].

[45] N. Bjerrum-Bohr, P.H. Damgaard, R. Monteiro and D. O'Connell, Algebras for amplitudes, JHEP 06 (2012) 061 [arXiv: 1203.0944] [INSPIRE].

[46] Z. Bern, L.J. Dixon, M. Perelstein and J. Rozowsky, Multileg one loop gravity amplitudes from gauge theory, Nucl. Phys. B 546 (1999) 423 [hep-th/9811140] [INSPIRE].

[47] Z. Bern, L.J. Dixon, D.C. Dunbar and D.A. Kosower, One loop $n$ point gauge theory amplitudes, unitarity and collinear limits, Nucl. Phys. B 425 (1994) 217 [hep-ph/9403226] [INSPIRE]. 
[48] Z. Bern, L.J. Dixon, D.C. Dunbar and D.A. Kosower, Fusing gauge theory tree amplitudes into loop amplitudes, Nucl. Phys. B 435 (1995) 59 [hep-ph/9409265] [INSPIRE].

[49] Z. Bern, L.J. Dixon, D. Dunbar, M. Perelstein and J. Rozowsky, On the relationship between Yang-Mills theory and gravity and its implication for ultraviolet divergences, Nucl. Phys. B 530 (1998) 401 [hep-th/9802162] [INSPIRE].

[50] Z. Bern, J. Carrasco, L.J. Dixon, H. Johansson, D. Kosower and R. Roiban, Three-loop superfiniteness of $N=8$ supergravity, Phys. Rev. Lett. 98 (2007) 161303 [hep-th/0702112] [INSPIRE].

[51] Z. Bern, J. Carrasco, L.J. Dixon, H. Johansson and R. Roiban, The ultraviolet behavior of $N=8$ supergravity at four loops, Phys. Rev. Lett. 103 (2009) 081301 [arXiv:0905.2326] [INSPIRE].

[52] S.J. Parke and T. Taylor, An amplitude for n-gluon scattering, Phys. Rev. Lett. 56 (1986) 2459 [INSPIRE].

[53] G. Georgiou, E.W.N. Glover and V.V. Khoze, Non-MHV tree amplitudes in gauge theory, JHEP 07 (2004) 048 [hep-th/0407027] [INSPIRE].

[54] J. Alwall, M. Herquet, F. Maltoni, O. Mattelaer and T. Stelzer, MadGraph 5: going beyond, JHEP 06 (2011) 128 [arXiv:1106.0522] [INSPIRE].

[55] C. Degrande, C. Duhr, B. Fuks, D. Grellscheid, O. Mattelaer and T. Reiter, UFO - The Universal FeynRules Output, Comput. Phys. Commun. 183 (2012) 1201 [arXiv:1108.2040] [INSPIRE].

[56] P. de Aquino, W. Link, F. Maltoni, O. Mattelaer and T. Stelzer, ALOHA: Automatic Libraries Of Helicity Amplitudes for Feynman Diagram Computations, Comput. Phys. Commun. 183 (2012) 2254 [arXiv:1108.2041] [INSPIRE].

[57] N.D. Christensen and C. Duhr, FeynRules - Feynman rules made easy, Comput. Phys. Commun. 180 (2009) 1614 [arXiv:0806.4194] [InSPIRE].

[58] J. Drummond and J. Henn, All tree-level amplitudes in N=4 SYM, JHEP 04 (2009) 018 [arXiv: 0808.2475] [INSPIRE].

[59] A. Hodges, Eliminating spurious poles from gauge-theoretic amplitudes, arXiv:0905.1473 [INSPIRE].

[60] V. Nair, A current algebra for some gauge theory amplitudes, Phys. Lett. B 214 (1988) 215 [INSPIRE].

[61] J. Broedel and J.J.M. Carrasco, Virtuous trees at five and six points for Yang-Mills and gravity, Phys. Rev. D 84 (2011) 085009 [arXiv:1107.4802] [INSPIRE].

[62] E. Plahte, Symmetry properties of dual tree-graph n-point amplitudes, Nuovo Cim. A 66 (1970) 713 [INSPIRE].

[63] R. Metsaev and A.A. Tseytlin, curvature cubed terms in string theory effective actions, Phys. Lett. B 185 (1987) 52 [inSPIRE].

[64] N. Bjerrum-Bohr, D.C. Dunbar, H. Ita, W.B. Perkins and K. Risager, MHV-vertices for gravity amplitudes, JHEP 01 (2006) 009 [hep-th/0509016] [INSPIRE].

[65] K. Risager, A direct proof of the CSW rules, JHEP 12 (2005) 003 [hep-th/0508206] [INSPIRE]. 
[66] H. Elvang, D.Z. Freedman and M. Kiermaier, Proof of the MHV vertex expansion for all tree amplitudes in $N=4$ SYM theory, JHEP 06 (2009) 068 [arXiv:0811.3624] [INSPIRE].

[67] H. Elvang and M. Kiermaier, Stringy KLT relations, global symmetries and $E_{7(7)}$ violation, JHEP 10 (2010) 108 [arXiv:1007.4813] [INSPIRE].

[68] P. van Nieuwenhuizen and C. Wu, On integral relations for invariants constructed from three Riemann tensors and their applications in quantum gravity, J. Math. Phys. 18 (1977) 182 [INSPIRE].

[69] Z. Bern, S. Davies, T. Dennen and Y.-t. Huang, Absence of three-loop four-point divergences in $N=4$ supergravity, Phys. Rev. Lett. 108 (2012) 201301 [arXiv:1202.3423] [INSPIRE].

[70] A.A. Tseytlin, Vector field effective action in the open superstring theory, Nucl. Phys. B 276 (1986) 391 [Erratum ibid. B 291 (1987) 876] [InSPIRE].

[71] M.B. Green and J.H. Schwarz, Supersymmetrical dual string theory. 2. Vertices and trees, Nucl. Phys. B 198 (1982) 252 [inSPIRE].

[72] J.H. Schwarz, Superstring theory, Phys. Rept. 89 (1982) 223 [INSPIRE].

[73] S. Stieberger and T.R. Taylor, Multi-gluon scattering in open superstring theory, Phys. Rev. D 74 (2006) 126007 [hep-th/0609175] [INSPIRE].

[74] A. Bilal, Higher derivative corrections to the nonAbelian Born-Infeld action, Nucl. Phys. B 618 (2001) 21 [hep-th/0106062] [INSPIRE].

[75] A.A. Tseytlin, On non-Abelian generalization of Born-Infeld action in string theory, Nucl. Phys. B 501 (1997) 41 [hep-th/9701125] [INSPIRE].

[76] S. Stieberger and T.R. Taylor, Complete six-gluon disk amplitude in superstring theory, Nucl. Phys. B 801 (2008) 128 [arXiv:0711.4354] [InSPIRE].

[77] O. Schlotterer and S. Stieberger, Motivic multiple zeta values and superstring amplitudes, arXiv:1205.1516 [INSPIRE]. 